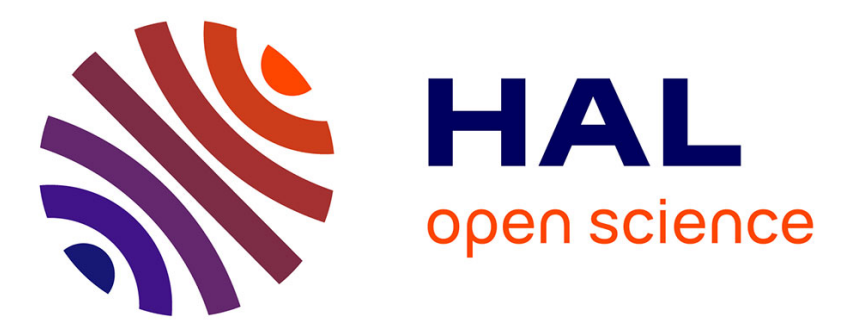

\title{
A virtual volume method for heterogeneous and anisotropic diffusion-reaction problems on general meshes.
}

Julien Coatléven

\section{- To cite this version:}

Julien Coatléven. A virtual volume method for heterogeneous and anisotropic diffusion-reaction problems on general meshes.. ESAIM: Mathematical Modelling and Numerical Analysis, 2017, 51 (3), pp.797-824. 10.1051/m2an/2016036 . hal-01581888

\section{HAL Id: hal-01581888 \\ https: / hal-ifp.archives-ouvertes.fr/hal-01581888}

Submitted on 5 Sep 2017

HAL is a multi-disciplinary open access archive for the deposit and dissemination of scientific research documents, whether they are published or not. The documents may come from teaching and research institutions in France or abroad, or from public or private research centers.
L'archive ouverte pluridisciplinaire HAL, est destinée au dépôt et à la diffusion de documents scientifiques de niveau recherche, publiés ou non, émanant des établissements d'enseignement et de recherche français ou étrangers, des laboratoires publics ou privés. 


\title{
A VIRTUAL VOLUME METHOD FOR HETEROGENEOUS AND ANISOTROPIC DIFFUSION-REACTION PROBLEMS ON GENERAL MESHES
}

\author{
Julien COATlÉven
}

\begin{abstract}
Starting from the recently introduced virtual element method, we construct new diffusion fluxes in two and three dimensions that give birth to symmetric, unconditionally coercive finite volume like schemes for the discretization of heterogeneous and anisotropic diffusion-reaction problems on general, possibly nonconforming meshes. Convergence of the approximate solutions is proved for general tensors and meshes. Error estimates are derived under classical regularity assumptions. Numerical results illustrate the performance of the scheme. The link with the original vertex approximate gradient scheme is emphasized.
\end{abstract}

Mathematics Subject Classification. 65N08, 65N12, 65N15.

Received October 23, 2015. Revised March 16, 2016. Accepted May 16, 2016.

\section{INTRODUCTION}

Finite volumes are very attractive methods in many scientific fields such as biology, plasma physics, hydrodynamics, oil reservoir simulation, etc. They allow to deal easily with the non linearities occurring in complex, coupled physical models while preserving relevant physical properties such as local mass conservation. Moreover, they remain quite easy to implement, even in a high performance computing (HPC) context, with several level of parallelization. However, the most basic, appreciated and widely used finite volume scheme, namely the two-point flux approximation (TPFA) scheme, has limited abilities to cope with diffusion operators on distorted meshes: to remain consistent, it requires a very strong orthogonality hypothesis on the mesh, even more difficult to satisfy in presence of anisotropy and strong heterogeneities (see [19]). Consequently, the range of admissible meshes is quite small, and too restrictive to tackle the more complex geometries industries are now considering (for instance structurally complex subsurface models for oil and gas exploration).

Due to the economic importance of this issue, a huge literature exists on the extension to general meshes of the classical TPFA finite volume method, thus we will not try to be exhaustive. For a recent review, we refer the reader to [16]. Multi-point flux approximations (MPFA, see [1-3]) are the natural generalization of the TPFA. They allow to construct consistent and cell-centered flux approximations on distorted meshes and lead to compact stencils, but suffer from conditional coercivity (see $[5,6]$ ). More recently, new finite volume methods using additional face unknowns (the hybrid finite volume scheme, see $[17,20,21]$ ) or vertex unknowns (the vertex approximate gradient schemes (VAG, see $[8,22-24]$ ) have been introduced. They allow to obtain both coercivity and consistency on quite general, possibly nonconforming meshes, for strongly heterogeneous

Keywords and phrases. Heterogeneous diffusion-reaction problems, finite volumes, general meshes, virtual element method.

1 IFP Énergies nouvelles, 1 et 4 avenue de Bois-Préau, 92852 Rueil-Malmaison, France. julien.coatleven@ifpen.fr 
and anisotropic tensors, and require only star-shaped cells (notice that for the hybrid scheme extensions to a larger class of meshes with non-star shaped cells but still with planar faces exists, see [27]). The formerly introduced mimetic finite differences (see $[10-12,15]$ ) can be unified, in their first order formulation, with hybrid finite volumes in a generalized context (see [18]), giving birth to the class of Hybrid Mixed Methods (HMM). These new finite volume schemes are, at some point, based on non conforming variational formulations of the diffusion operators, in this sense closing the gap between finite volume methods and Galerkin approximations like finite elements and discontinuous Galerkin methods $(\mathrm{dG})$. From this observation, even if what we seek are finite volume schemes, it is interesting to see to which extent modern Galerkin methods are able to cope with generic meshes. Of course, we are not trying here to describe the corresponding tremendous literature, but only to motivate our observations. The mimetic finite differences have recently been reformulated in the very promising virtual element method (VEM) (see $[7,14]$ ), a Galerkin method that can cope with almost any mesh, without even requiring star-shaped cells. The mimetic technology underlying the VEM seems to be the one, among Galerkin approaches, that allows to handle the widest class of meshes in the simplest manner ( $\mathrm{dG}$ methods also give access to a wide class of meshes, but are much more involved). Consequently it appears as a very interesting approach for overcoming the limitations of finite volume methods. However, as classical finite elements, the VEM for diffusion problems does not ensure local mass conservation as a finite volume scheme would, nor does it present an obvious diffusion flux-based formulation, which would simplify its extension to some important non-linear flow problems.

The method we present here is nothing but an attempt to merge the clever ideas of the VEM/mimetic and VAG approaches into the finite volume setting. We are going to construct finite volume diffusion fluxes using the VEM technology with the modification that we keep the usually discarded cell unknown, and use ideas from the VAG scheme to treat non-planar faces. Then, we approximate the reaction part following the stabilized mimetic approach of [7]. We show that this stabilization leads to introducing "reaction fluxes" between degrees of freedom. Doing so, the mimetic technology provides access to its extremely wide range of admissible meshes, with possibly very exotic, non necessarily star shaped cells. Meanwhile our modifications allow us to retrieve the local mass conservation property, as well as a balanced flux-based formulation. Then, the method formally possesses the same analytic form than classical finite volume methods, which means that its extension to more complex problems, not discussed here, can follow well establish lines.

The paper will be organized as follows: in a first section, we describe basic notations and our diffusion-reaction model problem. In a second section, we explain how to construct our new diffusion fluxes in dimension 2 and 3 , introducing at the same time the approximation spaces. Then, we will describe how the stabilized approximation of the reaction term leads to introducing reaction fluxes. Then, in a third section, we establish convergence of the method and provide the usual error estimates. In the last section, we present some numerical results in dimension 2 and 3, that illustrate the good behavior of the scheme, even for very exotic meshes.

Notice that the method we present is formally very close to VAG schemes when reduced to their most basic characteristics, i.e. finite volume schemes that uses both cell and vertex unknowns. However our construction of diffusion fluxes, particularly in dimension 3, is to our knowledge different from VAG constructions that has appeared in the literature and provides access to a much wider range of admissible meshes, in particular those with non star-shaped cells. The approximating space, related to VEM spaces, is also very different from those appearing in usual finite volume methods and leads to a new interpretation which we consider as probably as interesting as the method itself. Moreover the stabilization of the reaction part we introduce here is very different from the one introduced in the context of approximating multiphase-flow in porous media with the VAG scheme (see $[8,23,24]$ ). Here, our stabilization is incorporated in the fluxes, producing diffusion-reaction fluxes, very different from usual VAG diffusion only fluxes.

Also notice that our work could be sum up by saying that we are constructing an enhanced VEM approximation by keeping the classically discarded cell unknown, useless for consistency, of the first order VEM method and modifying the stabilization accordingly to recover local conservativity. With this point of view, the main contributions of the present work is to show how to extend the schemes to cells with non-planar faces and also 
to show that keeping this additional unknown is the key to make the resulting scheme a finite volume one, thus bringing the elegance and flexibility of the mimetic technology to the classical finite volume setting.

\section{Model PRoblem, notations}

As a model problem we consider a classical diffusion-reaction problem, set on an open bounded domain $\Omega$ subset of $\mathbb{R}^{d}, d \in \mathbb{N} \backslash\{0\}$, that reads:

$$
-\operatorname{div}(\Lambda \nabla u)+\beta u=f \quad \text { in } \Omega,
$$

We complement it with homogeneous Dirichlet boundary conditions, i.e.

$$
u=0 \text { on } \partial \Omega,
$$

where $\partial \Omega=\bar{\Omega} \backslash \Omega$ is the boundary of the domain $\Omega$, assumed to be at least Lipschitz continuous. The weak solution associated to $(2.1)-(2.2)$ is the unique $u \in H_{0}^{1}(\Omega)$ such that:

$$
\int_{\Omega} \Lambda \nabla u \nabla v+\beta u v=\int_{\Omega} f v \quad \forall v \text { in } H_{0}^{1}(\Omega)
$$

under the additional hypothesis that $f \in L^{2}(\Omega)$, that $\beta$ is a measurable function from $\Omega$ to $\mathbb{R}$, with $\beta(\boldsymbol{x}) \geq 0$ for a.e. $\boldsymbol{x} \in \Omega$, and that $\Lambda$ is a measurable functions from $\Omega$ to $\mathbb{M}_{d}(\mathbb{R})$, the set of $d \times d$ matrices, and is such that for almost every (a.e.) $\boldsymbol{x} \in \Omega, \Lambda(\boldsymbol{x})$ is symmetric, positive definite and there exists two strictly positive real numbers $\lambda_{*}, \lambda^{*}$ such that for a.e. $\boldsymbol{x} \in \Omega$ and for every $\boldsymbol{\xi} \in \mathbb{R}^{d}$ :

$$
\lambda_{*}|\boldsymbol{\xi}|^{2} \leq \Lambda(\boldsymbol{x}) \boldsymbol{\xi} \cdot \boldsymbol{\xi} \leq \lambda^{*}|\boldsymbol{\xi}|^{2} .
$$

\subsection{Description of the meshes}

From now on, we assume that the domain $\Omega$ is a bounded generalized polyhedral (i.e. with potentially non planar faces) subset of $\mathbb{R}^{d}$. To fix vocabulary, the mesh associated to $\Omega$ is described as follows:

Definition 2.1 (General nonconforming meshes). Let $\Omega$ be a generalized polyhedral, open bounded connected subset of $\mathbb{R}^{d}, d=2$ or 3 , and $\partial \Omega=\bar{\Omega} \backslash \Omega$ denotes its boundary. A mesh on $\Omega$, denoted by $\mathcal{M}$, is defined as a set $\mathcal{M}=(\mathcal{T}, \mathcal{F}, \mathcal{S F}, \mathcal{V}, \widetilde{\mathcal{V}}, \mathcal{E}, \widetilde{\mathcal{E}})$ where:

1. $\mathcal{T}$ is a finite family of connected open disjoint and generalized polygonal subsets of $\Omega$ (the cells of the mesh), such that $\bar{\Omega}=\cup_{K \in \mathcal{T}} \bar{K}$. For any $K \in \mathcal{T}$, we denote by $|K|$ the $d$-dimensional Lebesgue measure of $|K|$, and we assume that $|K|>0$. We denote by $\partial K=\bar{K} \backslash K$ the boundary of $K$, by $h_{K}$ the diameter of $K$, and by $\boldsymbol{x}_{K}$ the barycenter of $K$.

2. $\mathcal{F}$ is a finite family of disjoint subsets of $\bar{\Omega}$ (the faces of the mesh) such that, for all $\sigma \in \mathcal{F}$, its $d-1$ dimensional Lebesgue measure $|\sigma|$ is assumed to be strictly positive, and its diameter is denoted $h_{\sigma}$. We assume that for any $K \in \mathcal{T}$, there exists a subset $\mathcal{F}_{K}$ of $\mathcal{F}$ such that $\partial K=\cup_{\sigma \in \mathcal{F}_{K}} \bar{\sigma}$. Then, for any $\sigma \in \mathcal{F}$, we denote by $\mathcal{T}_{\sigma}=\left\{K \in \mathcal{T} \mid \sigma \in \mathcal{F}_{K}\right\}$ (the neighbors of $\sigma$ ), and assume that either $\mathcal{T}_{\sigma}$ has exactly one element, and then $\sigma \subset \partial \Omega$ (the set of these faces, called boundary faces, is denoted $\mathcal{F}_{\text {ext }}$ ), or $\mathcal{T}_{\sigma}$ has exactly two elements (the set of these faces, called interior faces, is denoted $\mathcal{F}_{\text {int }}$ ). For all $K \in \mathcal{T}$ and all $\sigma \in \mathcal{F}_{K}$, we denote by $\boldsymbol{n}_{K, \sigma}$ the unit normal vector to $\sigma$ outward to $K$. For any $\sigma \in \mathcal{F}$, we denote by $\boldsymbol{x}_{\sigma}$ its barycenter.

3. $\mathcal{V}$ is a family of points (the vertices of the mesh) such that for any $K \in \mathcal{T}$, and any subset $H_{K}$ of $\mathcal{F}_{K}$ with $\operatorname{card}\left(H_{K}\right) \geq d$, then $\cap_{\sigma \in H_{K}}=\emptyset$ or $\cap_{\sigma \in H_{K}}=s$, for some $s \in \mathcal{V}$. For all $s \in \mathcal{V}$, we denote $\mathcal{F}_{s}$ the set $\{\sigma \in \mathcal{F} \mid s \in \sigma\}$, and $\mathcal{T}_{s}$ the set of cells $\{K \in \mathcal{T} \mid s \in \bar{K}\}$. For all $K \in \mathcal{T}$, the set $\mathcal{V}_{K}$ stands for $\left\{s \in \mathcal{V} \mid K \in \mathcal{T}_{s}\right\}$, while for all $\sigma \in \mathcal{F}$, the set $\mathcal{V}_{\sigma}$ stands for $\left\{s \in \mathcal{V} \mid \sigma \in \mathcal{F}_{s}\right\}$. Conversely, $\mathcal{F}_{s}$ stands for the set $\left\{\sigma \in \mathcal{F} \mid s \in \mathcal{V}_{\sigma}\right\}$. For any $s \in \mathcal{V}$, the geometrical position of the vertex will be denoted $\boldsymbol{x}_{s}$. 
4. $\mathcal{E}$ is a family of segments (the edges of the mesh) such that, for all $e \in \mathcal{E}$, its Lebesgue measure $|e|$, which coincides with its diameter $h_{e}$, is assumed to be strictly positive, and there exists exactly two vertices $s_{e, 1}, s_{e, 2}$ of $\mathcal{V}$ such that $e=] s_{e, 1}, s_{e, 2}\left[\right.$. The subset $\left\{s_{e, 1}, s_{e, 2}\right\}$ of $\mathcal{V}$ is denoted $\mathcal{V}_{e}$. In dimension 2, we have $\mathcal{F}=\mathcal{E}$ while in dimension 3, for any $\sigma \in \mathcal{F}$, there exists a subset $\mathcal{E}_{\sigma}$ of $\mathcal{E}$ such that $\partial \sigma=\cup_{e \in \mathcal{E}_{\sigma}} \bar{e}$ and we have $\mathcal{E}=\cup_{\sigma \in \mathcal{F}} \mathcal{E}_{\sigma}$. For any $\sigma \in \mathcal{F}$ and any $e \in \mathcal{E}_{\sigma}$ in dimension 3, we denote $\boldsymbol{n}_{\sigma, e}$ the unit normal vector to $e$ outward to $\sigma$. For any $e \in \mathcal{E}$, we denote by $\boldsymbol{x}_{e}$ its barycenter. By construction, we have $\mathcal{V}_{\sigma}=\cup_{e \in \mathcal{E}_{\sigma}} \mathcal{V}_{e}$. We denote $\mathcal{E}_{\text {ext }}=\left\{e \in \mathcal{E} \mid \sigma \in \mathcal{F}_{\text {ext }}\right.$ with $\left.e \in \mathcal{E}_{\sigma}\right\}$, and $\mathcal{E}_{\text {int }}=\mathcal{E} \backslash \mathcal{E}_{\text {ext }}$

In dimension 3 , we need to complement the definition of faces to handle non-planar faces that are composed of several planar subfaces:

5. $\mathcal{S F}$ is a finite family of disjoint subsets of $\bar{\Omega}$ (the subfaces of the mesh) such that, for all $f \in \mathcal{F}, f$ is polygonal, included in a hyperplane of $\mathbb{R}^{d}$, and its Lebesgue measure $|f|$ is assumed to be strictly positive. We assume that for any $\sigma \in \mathcal{F}$, there exist a subset $\mathcal{S F}_{\sigma}$ of $\mathcal{S F}$ such that $\bar{\sigma}=\cup_{f \in \mathcal{S} \mathcal{F}_{\sigma}} \bar{f}$, and that $\mathcal{S} \mathcal{F}=\cup_{\sigma \in \mathcal{F}} \mathcal{S} \mathcal{F}_{\sigma}$. For all $K \in \mathcal{T}$, for all $\sigma \in \mathcal{F}_{K}$ and all $f \in \mathcal{S F}_{\sigma}$, we denote by $\boldsymbol{n}_{K, f}$ the constant unit normal vector to $f$ outward to $K$, and $\mathcal{S F}_{K}=\cup_{\sigma \in \mathcal{F}_{K}} \mathcal{S} \mathcal{F}_{\sigma}$. For any $f \in \mathcal{S} \mathcal{F}$, we denote by $\boldsymbol{x}_{f}$ its barycenter.

7. $\widetilde{\mathcal{E}}$ is a family of segments (the additional edges due to the subfaces) such that, for all $e \in \widetilde{\mathcal{E}}$, its Lebesgue measure $|e|$, which coincides with its diameter $h_{e}$, is assumed to be strictly positive, and $e \notin \mathcal{E}$. For any $f \in \mathcal{S F}$, there exists a subset $\widetilde{\mathcal{E}}_{f}$ of $\widetilde{\mathcal{E}}$ and a subset $\mathcal{E}_{f}$ of $\mathcal{E}$ such that $\partial f=\left(\cup_{f \in \widetilde{\mathcal{E}}_{f}} \bar{e}\right) \cup\left(\cup_{f \in \mathcal{E}_{f}} \bar{e}\right)$. For any $f \in \mathcal{S F}$ and any $e \in \widetilde{\mathcal{E}}_{f}$, we denote $\boldsymbol{n}_{f, e}$ the unit normal vector to $e$ outward to $f$ while for any $e \in \widetilde{\mathcal{E}}$, we denote by $\boldsymbol{x}_{e}$ its barycenter.

6. $\widetilde{\mathcal{V}}$ is a family of points (the additional vertices due to the subfaces) such that for any $s \in \widetilde{\mathcal{V}}$, there exists $\sigma \in \mathcal{F}$ and a subset $E_{\sigma}$ of $\cup_{f \in \mathcal{S} \mathcal{F}_{\sigma}} \widetilde{\mathcal{E}}_{f}$ such that $\cap_{e \in E_{\sigma}}=s$ and $s \notin \mathcal{V}$. For any $s \in \widetilde{\mathcal{V}}$, the geometrical position of the vertex will be denoted $\boldsymbol{x}_{s}$. For any $e \in \widetilde{\mathcal{E}}$, and there exists exactly two vertices $s_{e, 1}, s_{e, 2}$ of $\mathcal{V} \cup \widetilde{\mathcal{V}}$ such that $e=] s_{e, 1}, s_{e, 2}\left[\right.$. The subset of $\left\{s_{e, 1}, s_{e, 2}\right\}$ included in $\mathcal{V}$ is denoted $\mathcal{V}_{e}$, while the subset of $\left\{s_{e, 1}, s_{e, 2}\right\}$ included in $\widetilde{\mathcal{V}}$ is denoted $\widetilde{\mathcal{V}}_{e}$. For any $f \in \mathcal{S F}$, we denote $\mathcal{V}_{f}$ the set $\{s \in \mathcal{V} \mid s \in f\}$, and $\widetilde{\mathcal{V}}_{f}$ the set $\{s \in \widetilde{\mathcal{V}} \mid s \in f\}$. For any $\sigma \in \mathcal{F}$, we denote $\widetilde{\mathcal{V}}_{\partial \sigma, f}$ the subset of $\widetilde{\mathcal{V}}_{f}$ such that any $s \in \widetilde{\mathcal{V}}_{f}$ belongs to $\partial \sigma$, and $\widetilde{\mathcal{V}}_{\sigma, f}$ the subset of $\widetilde{\mathcal{V}}_{f}$ such that any $s \in \widetilde{\mathcal{V}}_{f}$ belongs to the interior of $\sigma$. We also denote $\widetilde{\mathcal{V}}_{\partial \sigma}=\cup_{f \in \mathcal{S} \mathcal{F}_{\sigma}} \widetilde{\mathcal{V}}_{\partial \sigma, f}$ and $\widetilde{\mathcal{V}}_{\sigma}=\cup_{f \in \mathcal{S} \mathcal{F}_{\sigma}} \widetilde{\mathcal{V}}_{\sigma, f}$. Finally, for any $s \in \mathcal{V} \cup \widetilde{\mathcal{V}}$, we denote $\mathcal{S F}_{s}$ the set $\left\{f \in \mathcal{S F} \mid s \in \mathcal{V}_{f} \cup \widetilde{\mathcal{V}}_{f}\right\}$.

In dimension 2 , we simply have $\mathcal{S F}=\mathcal{F}=\mathcal{E}, \widetilde{\mathcal{E}}=\emptyset$ and $\widetilde{\mathcal{V}}=\emptyset$. In dimension 3 , the last three sets describes how any face is decomposed into one or more planar subfaces, the face itself not being necessarily planar. In more classical definitions of general non conforming meshes, this decomposition is implicit and the elements of $\mathcal{S F}$ are directly called faces. However, this considerably increase the number of vertices and edges, as one has then to consider the sets $\mathcal{V} \cup \widetilde{\mathcal{V}}$ and $\mathcal{E} \cup \widetilde{\mathcal{E}}$. As we will construct our degrees of freedom on vertices, we choose to call faces the entire common boundary of any couple of cells, at the expense of a more involved construction of the numerical method. Notice that in practice, the set $\widetilde{\mathcal{V}}$ will very often be empty: in particular, in the case where the cells all generalized hexahedrons (i.e. hexahedrons whose points have been moved such that some faces are no longer planar), any face can be split into two triangular subfaces based on the existing points (see Fig. 1).

Notice that we do not have assumed that any element is star-shaped, nor did we assume that faces are planar in dimension 3: our definition is very permissive and corresponds to the most common practical situations: it covers in particular mesh deformations obtained by moving their vertices, very common in industrial applications.

In the following, we denote $h=\max _{K \in \mathcal{T}_{h}} h_{K}$. For any element $\mathcal{O}$, we denote by $\mathbb{P}_{k}(\mathcal{O})$ the space of polynomial functions of degree at most $k$ on $\mathcal{O}$, and by $\mathbb{M}_{k}(\mathcal{O})$ the space of monomials of degree exactly $k$ on $\mathcal{O}$. Moreover, we denote by $\pi_{k, \mathcal{O}}$ the $L^{2}$ orthogonal projector on $\mathbb{P}_{k}(\mathcal{O})$. We also denote $\forall u, v \in H^{1}(\Omega)$

$$
\mathcal{A}(\Lambda, \beta ; u, v)=a(\Lambda ; u, v)+r(\beta ; u, v), \quad a(\Lambda ; u, v)=(\Lambda \nabla u, \nabla v)_{0, \Omega}, \text { and } r(\beta ; u, v)=(\beta u, v)_{0, \Omega}
$$



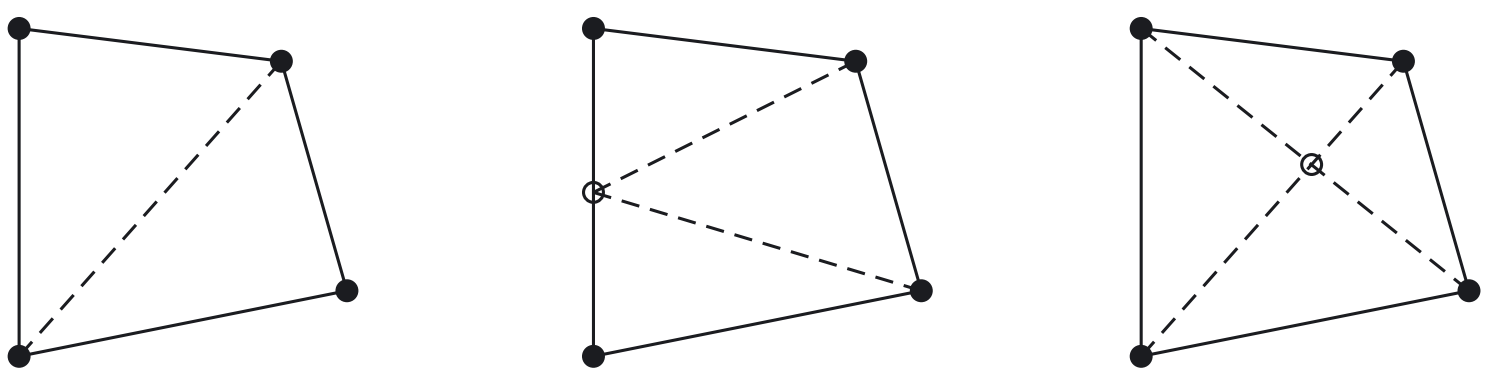

FIGURE 1. Examples of splitting for a non planar face $\sigma$ : left: $\widetilde{\mathcal{V}}_{\partial \sigma}=\widetilde{\mathcal{V}}_{\sigma}=\emptyset$, center: $\widetilde{\mathcal{V}}_{\partial \sigma} \neq \emptyset$ $\widetilde{\mathcal{V}}_{\sigma}=\emptyset$, right: $\widetilde{\mathcal{V}}_{\partial \sigma}=\emptyset, \widetilde{\mathcal{V}}_{\sigma} \neq \emptyset$, plane circles are the vertices of $\sigma$, empty circles are the additional vertices defining subfaces.

and $\forall u, v \in H^{1}(K)$

$$
\mathcal{A}^{K}(\Lambda, \beta ; u, v)=a^{K}(\Lambda ; u, v)+r^{K}(\beta ; u, v), \quad a(\Lambda ; u, v)=(\Lambda \nabla u, \nabla v)_{0, K}, \text { and } r^{K}(\beta ; u, v)=(\beta u, v)_{0, K}
$$

where $(\cdot, \cdot)_{0, \mathcal{O}}$ denote the $L^{2}(\mathcal{O})$ scalar product for any open set $\mathcal{O}$.

Definition 2.2. We say that a mesh $\mathcal{M}$ has optimal polynomial approximation properties if there exits $C_{\text {poly }}>$ 0 such that for any $K \in \mathcal{T}$ and any $v \in H^{s}(K)$, there exists $p \in \mathbb{P}_{k}(K)$, with $s-1 \leq k<s$, such that

$$
|v-p|_{H^{m}(K)} \leq C_{\text {poly }} h_{K}^{s-m}|v|_{H^{s}(K)} \quad \text { for } 0 \leq m \leq s
$$

In what follows, we will assume that our mesh satisfies:

(A1) There exists a real number $\rho>0$ and a matching simplicial submesh $\mathcal{S T}$ of $\mathcal{M}$ such that for any $T \in \mathcal{S} \mathcal{T}$, $\rho h_{T} \leq r_{T}$ where $r_{T}$ is the inradius of $T$, and for any $T \in \mathcal{T}$ and any $T \in \mathcal{S} \mathcal{T}$ such that $T \subset K, \rho h_{K} \leq h_{T}$.

Notice that assumption (A1) is not very restrictive: it simply requires that any cell of the mesh can itself be meshed by simplices. Any generalized polygon with piecewise planar faces obviously satisfies this assumption. It can be shown (see e.g. $[9,26]$ ) that these assumptions indeed imply optimal polynomial approximation properties.

\section{A virtual VOlume method}

Ultimately, we wish to describe any approximating function $v$ in any dimension simply by the following degrees of freedom:

- the mean value $v_{K}=\pi_{0, K}(v)$ of $v$ over each cell $K \in \mathcal{T}$,

- the values $v_{s}=v\left(\boldsymbol{x}_{s}\right)$ of $v$ at each vertex $s \in \mathcal{V}$.

The corresponding local set of dofs is denoted

$$
X(K)=\left\{v=\left(v_{K},\left(v_{s}\right)_{s \in \mathcal{V}_{K}}\right) \in \mathbb{R} \times \mathbb{R}^{\operatorname{card}\left(\mathcal{V}_{K}\right)}\right\}
$$

\subsection{Discrete spaces in dimension 2}

Following the construction of virtual element methods of [7], we start by defining a projector $\pi_{K}^{\nabla}: H^{1}(K) \longmapsto$ $\mathbb{P}_{1}(K)$ by:

$$
\mid \begin{aligned}
& \left.a^{K}\left(\pi_{0, K}(\Lambda) ; \pi_{K}^{\nabla} v, q\right)=-\int_{K} v \operatorname{div}\left(\pi_{0, K}(\Lambda) \nabla q\right)\right)+\int_{\partial K} v \pi_{0, K}(\Lambda) \nabla q \cdot \boldsymbol{n}_{\partial K} \quad \forall q \in \mathbb{P}_{1}(K) \\
& \frac{1}{|K|} \int_{K} \pi_{K}^{\nabla} v=\frac{1}{|K|} \int_{K} v
\end{aligned}
$$


where $\boldsymbol{n}_{\partial K}$ denotes the unit normal vector to $\partial K$ outward to $K$. Denoting $\Lambda_{K}=\pi_{0, K}(\Lambda)$, the expression of $a^{K}\left(\pi_{K}^{\nabla} v, q\right)$ (since $\left.\operatorname{div}\left(\pi_{0, K}(\Lambda) \nabla q\right)\right)=\operatorname{div}\left(\Lambda_{K} \nabla q\right)=0$ for any $\left.q \in \mathbb{P}_{1}(K)\right)$ can even be simplified for any $v \in H^{1}(K)$ in

$$
a^{K}\left(\Lambda_{K} ; \pi_{K}^{\nabla} v, q\right)=\int_{\partial K} v \Lambda_{K} \nabla q \cdot \boldsymbol{n}_{\partial K} \quad \forall q \in \mathbb{P}_{1}(K)
$$

or, equivalently

$$
a^{K}\left(\Lambda_{K} ; \pi_{K}^{\nabla} v, q\right)=\sum_{\sigma \in \mathcal{F}_{K}} \int_{\sigma}\left(v-v_{K}\right) \Lambda_{K} \nabla q \cdot \boldsymbol{n}_{\partial K} \quad \forall q \in \mathbb{P}_{1}(K) .
$$

Then, for any $K \in \mathcal{T}$, we consider the space:

$$
B_{1}(\partial K)=\left\{v \in C^{0}(\partial K) \mid v_{\left.\right|_{\sigma}} \in \mathbb{P}_{1}(\sigma), \forall \sigma \subset \partial K\right\}
$$

Obviously, $B_{1}(\partial K)$ is a space of dimension $\operatorname{card}\left(\mathcal{F}_{K}\right)=\operatorname{card}\left(\mathcal{V}_{K}\right)$, as any continuous function on $\partial K$ which is linear on each face (which coincides with edges in dimension 2) is uniquely determined by its values at the vertices. With this space, we can consider the finite dimensional space $\widetilde{V}(K)$ defined as:

$$
\widetilde{V}(K)=\left\{v \in H^{1}(K) \mid v_{\left.\right|_{\partial K}} \in B_{1}(\partial K) \text { and }-\operatorname{div}(\Lambda \nabla v) \in \mathbb{P}_{1}(K)\right\}
$$

Now, instead of considering the space $V(K)$ described in [14], we follow the alternative approach of [7] and consider the space

$$
W(K)=\left\{w \in \tilde{V}(K) \mid\left(w-\pi_{K}^{\nabla} w, q\right)_{0, K}=0, \forall q \in \mathbb{M}_{1}(K)\right\}
$$

for which the projector $\pi_{K}^{\nabla}$ coincides with the classical $L^{2}$ orthogonal projector $\pi_{1, K}$ on $\mathbb{P}_{1}(K)$ by construction. Notice that we did not enforce $\left(w-\pi_{K}^{\nabla} w, q\right)_{0, K}=0$ for the monomial of $\mathbb{M}_{0}(K)$, as is the case in [7]. This is due to the fact that our additional cell dof takes automatically care of this constant part through the definition of $\pi_{K}^{\nabla}$. The key idea behind this space is the fact that the operator $\pi_{K}^{\nabla}$ can be directly computed from the degrees of freedom for elements of $W(K)$, which then leads to a straightforward approximation of the diffusion term. The corresponding global space is:

$$
W=\left\{w \in H^{1} \mid w_{\mid K} \in W(K)\right\}
$$

In the same way as in [7], we have:

Proposition 3.1. The degrees of freedom of $X(K)$ are unisolvent for $W(K)$.

Proof. The proof is identical to the proof given in [7] for $W(K)$. As we detail the proof in the more complex case of dimension 3, we refer the reader to either [7] or Proposition 3.5 for more details.

Still following the idea of the VEM, at this point we consider an approximation of the bilinear form $a(\cdot, \cdot)$ for functions in $W(K)$ by combining $a(\cdot, \cdot)$ and $\pi_{K}^{\nabla}$ and adding a stabilization term for the $H^{1}$ norm:

$$
a_{h}(u, v)=\sum_{K \in \mathcal{T}} a_{h}^{K}(u, v) \quad a_{h}^{K}(u, v)=a^{K}\left(\Lambda_{K} ; \pi_{K}^{\nabla} u, \pi_{K}^{\nabla} v\right)+s^{K}\left(u-\pi_{K}^{\nabla}(u), v-\pi_{K}^{\nabla}(v)\right) \quad \forall(u, v) \in W(K)
$$

The bilinear form $s^{K}(\cdot, \cdot)$, added to ensure the coercivity of the resulting scheme, is given by

$$
s^{K}(u, v)=h_{K}^{d-2} \sum_{s \in \mathcal{V}_{K}} \sum_{s^{\prime} \in \mathcal{V}_{K}} S_{K, s, s^{\prime}} u\left(\boldsymbol{x}_{s}\right) v\left(\boldsymbol{x}_{s^{\prime}}\right) \quad \forall(u, v) \in W(K)
$$

and $S_{K}=\left(S_{K, s, s^{\prime}}\right)_{s, s^{\prime} \in \mathcal{V}_{K}}$ can be any symmetric positive definite matrix independent on $h$. As for any $q \in$ $\mathbb{P}_{1}(K)$, we have $\pi_{K}^{\nabla}(q)=q$, the consistency property:

$$
a_{h}^{K}(u, q)=a^{K}\left(\Lambda_{K} ; u, q\right) \quad \forall u \in H^{1}(K), \forall q \in \mathbb{P}_{1}(K)
$$


is obtained by construction. Notice that this stabilization says a priori nothing on the cell degree of freedom. However, for any $v \in W(K)$, we get $\pi_{0, K}(v)=\pi_{0, K}\left(\pi_{K}^{\nabla}(v)\right)$, so the a priori missing term is in fact zero on $W(K)$. Following [13,14], it is clear that the scaling $h_{K}^{d-2}$ of the stabilization terms ensures that:

Lemma 3.2. There exists $\alpha_{*}>0$ and $\alpha^{*}>0$, depending on $\rho$ but independent on $h$, such that for any $(u, v) \in W(K)^{2}$, we have

$$
a_{h}^{K}(u, v) \leq \alpha^{*} \mid\|\nabla u\|_{L^{2}(K)}\|\nabla v\|_{L^{2}(K)}
$$

and for any $u \in W(K)$

$$
\alpha_{*}\|\nabla u\|_{L^{2}(K)}^{2} \leq a_{h}^{K}(u, u)
$$

\subsection{Diffusion fluxes in dimension 2}

To obtain diffusion fluxes, we have to rewrite a little the bilinear form $a$ on each cell $K$. Using again the fact that the gradients $\pi_{K}^{\nabla} v$ and $\nabla q$ are constant over $K$, we can give an explicit expression for $\pi_{K}^{\nabla}$ for elements of $W(K)$. Indeed, we have:

$$
\int_{K} \Lambda_{K} \nabla \pi_{K}^{\nabla} v \nabla q=\sum_{\sigma \in \mathcal{F}_{K}} \int_{\sigma}\left(v-v_{K}\right) \Lambda_{K} \nabla q \cdot \boldsymbol{n}_{\partial K} .
$$

As $\Lambda_{K}, \nabla \pi_{K}^{\nabla} v$ and $\nabla q$ are constant over $K$, and $\Lambda_{K}$ is invertible, we deduce that:

$$
\nabla \pi_{K}^{\nabla} v=\frac{1}{|K|} \sum_{\sigma \in \mathcal{F}_{K}} \int_{\sigma}\left(v-v_{K}\right) \boldsymbol{n}_{K, \sigma},
$$

and finally, using the fact that $v$ is linear on each edge/face $\sigma$, we get:

$$
\nabla \pi_{K}^{\nabla} v=\sum_{\sigma \in \mathcal{F}_{K}} \frac{|\sigma|}{2|K|} \sum_{s \in \mathcal{V}_{\sigma}}\left(v_{s}-v_{K}\right) \boldsymbol{n}_{K, \sigma} .
$$

Denoting

$$
\boldsymbol{g}_{K, s}=\sum_{\sigma \in \mathcal{F}_{s} \cap \mathcal{F}_{K}} \frac{|\sigma|}{2|K|} \boldsymbol{n}_{K, \sigma}
$$

we get

$$
\nabla \pi_{K}^{\nabla} v=\sum_{s \in \mathcal{V}_{K}}\left(v_{s}-v_{K}\right) \boldsymbol{g}_{K, s} \quad \text { and } \quad \pi_{K}^{\nabla} v=v_{K}+\sum_{s \in \mathcal{V}_{K}}\left(v_{s}-v_{K}\right) \boldsymbol{g}_{K, s} \cdot\left(\boldsymbol{x}-\boldsymbol{x}_{K}\right)
$$

Then, as $\forall(u, v) \in W(K)$

$$
\mathcal{A}_{h}^{K}(u, v)=|K| \Lambda_{K} \nabla \pi_{K}^{\nabla}(u) \pi_{K}^{\nabla}(v)+h_{K}^{d-2} \sum_{s \in \mathcal{V}_{K}} \sum_{s^{\prime} \in \mathcal{V}_{K}} S_{K, s, s^{\prime}}\left(u\left(\boldsymbol{x}_{s}\right)-\pi_{K}^{\nabla}(u)\left(\boldsymbol{x}_{s}\right)\right)\left(v\left(\boldsymbol{x}_{s^{\prime}}\right)-\pi_{K}^{\nabla}(v)\left(\boldsymbol{x}_{s}\right)\right)
$$

using the explicit formula for $\pi_{K}^{\nabla}$ and expanding everything, we get, after a straightforward computation:

$$
a_{h}^{K}(u, v)=\sum_{s \in \mathcal{V}_{K}} \sum_{s^{\prime} \in \mathcal{V}_{K}} \mathbb{A}_{K}^{s, s^{\prime}}\left(u_{s}-u_{K}\right)\left(v_{s^{\prime}}-v_{K}\right)
$$

where

$$
\mid \begin{aligned}
& \mathbb{A}_{K}^{s, s^{\prime}}=|K| \Lambda_{K} \boldsymbol{g}_{K, s} \cdot \boldsymbol{g}_{K, s^{\prime}}+h_{K}^{d-2} \mathbb{S}_{K}^{s, s^{\prime}}, \\
& \mathbb{S}_{K}^{s, s^{\prime}}=y_{K, s^{\prime}}^{T} S_{K} y_{K, s}, \\
& y_{K, s}=\left(y_{K, s}^{s \prime}\right)_{s^{\prime \prime} \in \mathcal{V}_{K}}, \quad y_{K, s}^{s^{\prime \prime}}=\delta_{s, s^{\prime \prime}}-\boldsymbol{g}_{K, s} \cdot\left(\boldsymbol{x}_{s^{\prime \prime}}-\boldsymbol{x}_{K}\right)
\end{aligned}
$$


where $\delta_{s, s^{\prime \prime}}$ is the Kronecker symbol. The fluxes are then defined by setting

$$
F_{K, s}(u)=\sum_{s^{\prime} \in \mathcal{V}_{K}} \mathbb{A}_{K}^{s^{\prime}, s}\left(u_{K}-u_{s^{\prime}}\right)
$$

in order to finally obtain:

$$
a_{h}(u, v)=\sum_{K \in \mathcal{T}} \sum_{s \in \mathcal{V}_{K}} F_{K, s}(u)\left(v_{K}-v_{s}\right)
$$

Remark 3.3. In dimension 2 , the flux $F_{K, s}$ we constructed are very close to those of the original VAG scheme of [22], the difference being the stabilization term. Undoubtedly, proceeding along the same lines as [18], both schemes could be unified at least formally in the same framework by allowing a matrix as stabilization parameter in the VAG scheme. However, the derivation from mimetic techniques we propose here is different and appears as very interesting in itself. In particular it allows an interpretation of the scheme as a variational approximation using the space $W(K)$ while allowing to handle more general meshes, in particular those with non star-shaped cells.

\subsection{Discrete spaces in dimension 3}

In dimension 3 , still following the ideas of $[7,14]$, we have to introduce an intermediate approximation space on planar faces before defining its counterpart on cells, if we wish to keep the same set of degrees of freedom. Consequently, we start by defining a projector $\pi_{f}^{\nabla}: H^{1}(f) \longmapsto \mathbb{P}_{1}(f)$ by setting:

$$
\mid \begin{aligned}
& \left(\nabla \pi_{f}^{\nabla} v, \nabla q\right)_{0, f}=-\int_{f} v \Delta q+\int_{\partial f} v \nabla q \cdot \boldsymbol{n}_{\partial f} \quad \forall q \in \mathbb{P}_{1}(f) \\
& \frac{1}{|\partial f|} \int_{\partial f} \pi_{f}^{\nabla} v=\frac{1}{|\partial f|} \int_{\partial f} v
\end{aligned}
$$

Again, the above expression can be simplified into:

$$
\left(\nabla \pi_{f}^{\nabla}, v \nabla q\right)_{0, f}=\int_{\partial f} v \nabla q \cdot \boldsymbol{n}_{\partial f} \quad \forall q \in \mathbb{P}_{1}(f) .
$$

We start by considering the space $\widetilde{V}(f)$, defined by:

$$
\widetilde{V}(f)=\left\{v \in H^{1}(f) \mid v_{\left.\right|_{\partial f}} \in B_{1}(\partial f) \text { and } \Delta v \in \mathbb{P}_{1}(f)\right\} .
$$

Clearly, a reasoning similar to the proof of Proposition 3.1 shows that the elements of $\widetilde{V}(f)$ are uniquely defined by their their zero and first order moments on $f$ and their values at the vertices of $f$, which means some vertices of $\mathcal{V}_{\sigma}$ and some of the additional vertices of $\widetilde{\mathcal{V}}_{\partial \sigma} \cup \widetilde{\mathcal{V}}_{\sigma}$. However, we do not wish to add degrees of freedom related to those additional vertices. To this aim, we define the following space, which still contains the traces of elements of $\mathbb{P}_{1}(K)$ for any $K \in \mathcal{T}_{\sigma}$ :

$$
\widetilde{V}(\sigma)=\left\{v \in H^{1}(\sigma) \mid v_{\left.\right|_{f}} \in \tilde{V}(f) \quad, \forall f \in \mathcal{S F}_{\sigma}, \text { and } v_{\left.\right|_{\partial \sigma}} \in B_{1}(\partial \sigma)\right\} .
$$

On this space values at vertices of $\widetilde{\mathcal{V}}_{\partial \sigma}$ do not add any degree of freedom: more precisely, for any $s \in \widetilde{\mathcal{V}}_{\partial \sigma}$, there exists $e \in \mathcal{E}_{\sigma}$ and two real numbers $\rho_{e, s, s_{e_{1}}}$ and $\rho_{e, s, s_{e_{2}}}$ such that $s$ belongs to the interior of $e, \mathcal{V}_{e}=\left\{s_{e_{1}}, s_{e_{2}}\right\}$ and for any $u \in \widetilde{V}(\sigma)$, we have:

$$
u\left(\boldsymbol{x}_{s}\right)=\rho_{e, s, s_{e_{1}}} u\left(\boldsymbol{x}_{s_{e_{1}}}\right)+\rho_{e, s, s_{e_{2}}} u\left(\boldsymbol{x}_{s_{e_{2}}}\right)
$$


and

$$
\boldsymbol{x}_{s}=\rho_{e, s, s_{e_{1}}} \boldsymbol{x}_{s_{e_{1}}}+\rho_{e, s, s_{e_{2}}} \boldsymbol{x}_{s_{e_{2}}} \quad \rho_{e, s, s_{e_{1}}}+\rho_{e, s, s_{e_{2}}}=1
$$

as $u$ is linear on $e$. However, this is not the case for values associated with vertices of $\widetilde{\mathcal{V}}_{\sigma}$. By analogy, and inspired by [22], we associate to each element $s$ of $\widetilde{\mathcal{V}}_{\sigma}$ a set of real numbers $\left(\rho_{s, \sigma, s^{\prime}}\right)_{s^{\prime} \in \mathcal{V}_{\sigma}}$ such that at most $d+1$ of them are non zeros, and:

$$
\boldsymbol{x}_{s}=\sum_{s^{\prime} \in \mathcal{V}_{\sigma}} \rho_{\sigma, s, s^{\prime}} \boldsymbol{x}_{s^{\prime}} \quad \sum_{s^{\prime} \in \mathcal{V}_{\sigma}} \rho_{\sigma, s, s^{\prime}}=1 .
$$

Clearly, if $\mathcal{T}_{\sigma}=\{K, L\}$ (respectively if $\mathcal{T}_{\sigma}=\{K\}$ ), we have for any $u \in \mathbb{P}_{1}(K \cup L)$ (respectively $u \in \mathbb{P}_{1}(K)$ )

$$
u\left(\boldsymbol{x}_{s}\right)=\sum_{s^{\prime} \in \mathcal{V}_{\sigma}} \rho_{\sigma, s, s^{\prime}} u\left(\boldsymbol{x}_{s^{\prime}}\right) .
$$

Thus, this barycentric interpolation is exact for traces on $\sigma$ of first order polynomials. The corresponding simplified trace space is thus defined as

$$
\hat{V}(\sigma)=\left\{v \in \widetilde{V}(\sigma) \mid v\left(\boldsymbol{x}_{s}\right)=\sum_{s^{\prime} \in \mathcal{V}_{\sigma}} \rho_{\sigma, s, s^{\prime}} v\left(\boldsymbol{x}_{s^{\prime}}\right) \forall s \in \widetilde{\mathcal{V}}_{\sigma}\right\} .
$$

As remarked when we described meshes, the sets $\widetilde{\mathcal{V}}_{\partial \sigma}$ are very often empty, and the sets $\widetilde{\mathcal{V}}_{\sigma}$ even more often. However we wished to allow complicated face decompositions, in particular to cover the case when one use a classical meshing algorithm to generate subfaces, which may require those additional points in complex situations.

Finally, our space on faces is defined as:

$$
W(\sigma)=\left\{w \in \hat{V}(\sigma) \mid \int_{f}\left(w-\pi_{f}^{\nabla} w\right) q=0 \forall q \in \mathbb{M}_{0}(f) \cup \mathbb{M}_{1}(f), \forall f \in \mathcal{S} \mathcal{F}_{\sigma}\right\}
$$

which imposes that each projector $\pi_{f}^{\nabla}$ coincides with the classical $L^{2}$ orthogonal projector $\pi_{1, f}$ on $\mathbb{P}_{1}(f)$. Obviously, we have:

Lemma 3.4. Any element of $W(\sigma)$ is uniquely defined by its values at the vertices of $\sigma$.

Proof. The proof is identical to the proof given in [7] for $W(K)$. For any $f \in \mathcal{S T}_{\sigma}$, we define the operator $\Delta_{0, f}^{-1}: L^{2}(f): \longrightarrow H^{1}(k)$ by setting for any $q \in L^{2}(f) \Delta_{0, f}^{-1} q=w$, where

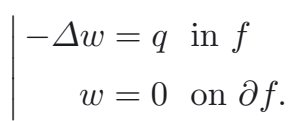

Then, we denote $R: L^{2}(f) \longrightarrow L^{2}(f)$ the operator defined by $R q=\pi_{1, f}\left(\Delta_{0, f}^{-1}(q)\right)$. Now, assume that the degrees of freedom of $u \in W(\sigma)$ and $v \in W(\sigma)$ are identical. By construction, if $u$ and $v$ are equal at each $s$ of $\mathcal{V}_{\sigma}$, we have $u=v$ on $\partial f$ for any $f \in \mathcal{S F}_{\sigma}$ and thus by definition $u-v$ satisfies (3.22) on each $f$ for some $q_{f} \in \mathbb{P}_{1}(f)$. Then, as for $u$ and $v$ in $W(\sigma)$ and any $f \in \mathcal{S T}_{\sigma}, \pi_{f}^{\nabla}(u)$ and $\pi_{f}^{\nabla}(v)$ solely depend on their values at the vertices of $\mathcal{V}_{\sigma}$, we deduce that $\pi_{f}^{\nabla}(u)=\pi_{f}^{\nabla}(v)$ for any $f \in \mathcal{S} \mathcal{T}_{\sigma}$. But by definition of $W(\sigma)$, this implies that for any $p \in \mathbb{P}_{1}(f)$ :

$$
(u-v, p)_{0, f}=\left(\pi_{1, f}(u-v), p\right)_{0, f}=\left(\pi_{f}^{\nabla}(u)-\pi_{f}^{\nabla}(v), p\right)_{0, f}=0 .
$$

Thus we have:

$$
\left(R q_{f}, q_{f}\right)_{0, f}=\left(\pi_{1, f}(u-v), q_{f}\right)_{0, f}=\left(u-v, q_{f}\right)_{0, f}=(\nabla(u-v), \nabla(u-v))_{L^{2}(f)}=0 .
$$

which immediately implies $u=v$ on any $f \in \mathcal{S T}_{\sigma}$ and thus on $\sigma$. 
Now we are in position to construct an approximation space on cells that can handle non-planar faces. We start by setting:

$$
\widetilde{V}(K)=\left\{v \in H^{1}(K) \mid v_{\left.\right|_{\sigma}} \in W(\sigma) \forall \sigma \in \mathcal{F}_{K} \text { and }-\operatorname{div}(\Lambda \nabla v) \in \mathbb{P}_{1}(K)\right\} .
$$

The three dimensional operator $\pi_{K}^{\nabla}: H^{1}(K) \longmapsto \mathbb{P}_{1}(K)$ is defined by, after the simplification of the volumic term we are used to now:

$$
\mid \begin{aligned}
& a^{K}\left(\Lambda_{K} ; \pi_{K}^{\nabla} v, q\right)=\int_{\partial K} v \Lambda_{K} \nabla q \cdot \boldsymbol{n}_{\partial K}=\int_{\partial K}\left(v-v_{K}\right) \Lambda_{K} \nabla q \cdot \boldsymbol{n}_{\partial K} \quad \forall q \in \mathbb{P}_{1}(K) \\
& \frac{1}{|K|} \int_{K} \pi_{K}^{\nabla} v=\frac{1}{|K|} \int_{K} v .
\end{aligned}
$$

Then, the approximation space is naturally:

$$
W(K)=\left\{v \in \widetilde{V}(K) \mid\left(v-\pi_{K}^{\nabla} v, q\right)_{0, K}=0, \forall q \in \mathbb{M}_{1}(K)\right\},
$$

the associated global space is

$$
W=\left\{v \in H^{1} \mid v_{\left.\right|_{K}} \in W(K) \forall K \in \mathcal{T}\right\}
$$

Proposition 3.5. The degrees of freedom of $X(K)$ are unisolvent for $W(K)$.

Proof. The proof is again identical to the proof given in [7] for $W(K)$. We define the operator $\Delta_{0, K, \Lambda}^{-1}$ : $L^{2}(K): \longrightarrow H^{1}(k)$ by setting for any $q \in L^{2}(K) \Delta_{0, K}^{-1} q=w$, where

$$
\begin{aligned}
-\operatorname{div}\left(\Lambda_{K} \nabla w\right)=q & \text { in } K \\
w=0 & \text { on } \partial K .
\end{aligned}
$$

Then, we denote $R: L^{2}(K) \longrightarrow L^{2}(K)$ the operator defined by $R q=\pi_{1, K}\left(\Delta_{0, K, \Lambda}^{-1}(q)\right)$. Now, assume that the degrees of freedom of $u \in W(K)$ and $v \in W(K)$ are identical. Then $u-v$ satisfies $\pi_{0, K}(u-v)=0$ and (3.27) for some $q \in \mathbb{P}_{1}(K)$. As for $u$ and $v$ in $W(K), \pi_{K}^{\nabla}(u)$ and $\pi_{K}^{\nabla}(v)$ solely depend on their values at the vertices and their mean value, we deduce that $\pi_{K}^{\nabla}(u)=\pi_{K}^{\nabla}(v)$. But by definition of $W(K)$, this implies that for any $p \in \mathbb{P}_{1}(K)$ :

$$
(u-v, p)_{0, K}=\left(\pi_{1, K}(u-v), p\right)_{0, K}=\left(\pi_{K}^{\nabla}(u)-\pi_{K}^{\nabla}(v), p\right)_{0, K}=0 .
$$

By construction, we then have:

$$
(R q, q)_{0, K}=\left(\pi_{1, K}(u-v), q\right)_{0, K}=(u-v, q)_{0, K}=\left(\Lambda_{K} \nabla(u-v), \nabla(u-v)\right)_{L^{2}(K)}=0
$$

which immediately implies $u=v$.

We define our approximation of the diffusive part as

$$
a_{h}(u, v)=\sum_{K \in \mathcal{T}} a_{h}^{K}(u, v) \quad a_{h}^{K}(u, v)=a^{K}\left(\Lambda_{K} ; \pi_{K}^{\nabla} u, \pi_{K}^{\nabla} v\right)+s^{K}\left(u-\pi_{K}^{\nabla}(u), v-\pi_{K}^{\nabla}(v)\right), \quad \forall(u, v) \in H^{1}(K)
$$

where the bilinear form $s^{K}(\cdot, \cdot)$, added to ensure the coercivity of the resulting scheme, is again given by

$$
s^{K}(u, v)=h_{K}^{d-2} \sum_{s \in \mathcal{V}_{K}} \sum_{s^{\prime} \in \mathcal{V}_{K}} S_{K, s, s^{\prime}} u\left(\boldsymbol{x}_{s}\right) v\left(\boldsymbol{x}_{s^{\prime}}\right) \quad \forall(u, v) \in W(K)
$$

with $S_{K}=\left(S_{K, s, s^{\prime}}\right)_{s, s^{\prime} \in \mathcal{V}_{K}}$ can be any symmetric positive definite matrix independent on $h$. For any $q \in \mathbb{P}_{1}(K)$, $\pi_{K}^{\nabla}(q)=q$ implies again by construction the consistency property:

$$
a_{h}^{K}(u, q)=a^{K}\left(\Lambda_{K} ; u, q\right) \quad \forall u \in H^{1}(K), \forall q \in \mathbb{P}_{1}(K) .
$$


Exactly as in dimension 2, we have

Lemma 3.6. There exists $\alpha_{*}>0$ and $\alpha^{*}>0$, depending on $\rho$ but independent on $h$, such that for any $(u, v) \in W(K)^{2}$, we have

$$
a_{h}^{K}(u, v) \leq \alpha^{*}|| \nabla u\left\|_{L^{2}(K)}\right\| \nabla v \|_{L^{2}(K)}
$$

and for any $u \in W(K)$

$$
\alpha_{*}\|\nabla u\|_{L^{2}(K)}^{2} \leq a_{h}^{K}(u, u)
$$

\subsection{Diffusion fluxes in dimension 3}

For any $v \in W(K)$, and any edge $e \in \mathcal{E}_{K} \cup \widetilde{\mathcal{E}}_{K}$, we have $v \in \mathbb{P}_{1}(e)$, thus, as $\sum_{s \in \mathcal{V}_{e} \cup \widetilde{\mathcal{V}}_{e}} \boldsymbol{n}_{e, s}=0$ :

$$
v_{\left.\right|_{e}}=\sum_{s \in \mathcal{V}_{e} \cup \widetilde{\mathcal{V}}_{e}} \frac{v_{s}}{2}+\frac{1}{|e|} \sum_{s \in \mathcal{V}_{e} \cup \widetilde{\mathcal{V}}_{e}}\left(v_{s}-v_{K}\right) \boldsymbol{n}_{e, s} \cdot\left(\boldsymbol{x}-\boldsymbol{x}_{e}\right) \quad \frac{1}{|e|} \int_{e} v=\sum_{s \in \mathcal{V}_{e} \cup \widetilde{\mathcal{V}}_{e}} \frac{v_{s}}{2} .
$$

Then, for any $f \in \mathcal{S} \mathcal{F}_{K}$, we easily get that:

$$
\nabla \pi_{f}^{\nabla}(v)=\sum_{e \in \mathcal{E}_{f} \cup \widetilde{\mathcal{E}}_{f}} \sum_{s \in \mathcal{V}_{e} \cup \widetilde{\mathcal{V}}_{e}} \frac{|e|}{2|f|}\left(v_{s}-v_{K}\right) \boldsymbol{n}_{f, e}=\sum_{s \in \mathcal{V}_{f} \cup \widetilde{\mathcal{V}}_{f}} \sum_{e \in\left(\mathcal{E}_{f} \cup \widetilde{\mathcal{E}}_{f}\right) \cap\left(\mathcal{E}_{s} \cup \widetilde{\mathcal{E}}_{s}\right)} \frac{|e|}{2|f|}\left(v_{s}-v_{K}\right) \boldsymbol{n}_{f, e}
$$

and

$$
\frac{1}{|\partial f|} \int_{f} v=\sum_{e \in \mathcal{E}_{f} \cup \widetilde{\mathcal{E}}_{f}} \sum_{s \in \mathcal{V}_{e} \cup \widetilde{\mathcal{V}}_{e}} \frac{|e|}{2|\partial f|} v_{s}=\sum_{s \in \mathcal{V}_{f} \cup \widetilde{\mathcal{V}}_{f}} \sum_{e \in\left(\mathcal{E}_{f} \cup \widetilde{\mathcal{E}}_{f}\right) \cap\left(\mathcal{E}_{s} \cup \widetilde{\mathcal{E}}_{s}\right)} \frac{|e|}{2|\partial f|} v_{s}
$$

Denoting

$$
\boldsymbol{g}_{f, s}=\sum_{e \in\left(\mathcal{E}_{f} \cup \widetilde{\mathcal{E}}_{f}\right) \cap\left(\mathcal{E}_{s} \cup \widetilde{\mathcal{E}}_{s}\right)} \frac{|e|}{2|f|} \boldsymbol{n}_{f, e} \quad \text { and } \quad m_{f, s}=\sum_{e \in\left(\mathcal{E}_{f} \cup \widetilde{\mathcal{E}}_{f}\right) \cap\left(\mathcal{E}_{s} \cup \widetilde{\mathcal{E}}_{s}\right)} \frac{|e|}{2|\partial f|}
$$

we get:

$$
\pi_{f}^{\nabla} v=\sum_{s \in \mathcal{V}_{f} \cup \widetilde{\mathcal{V}}_{f}} m_{f, s} v_{s}+\sum_{s \in \mathcal{V}_{f} \cup \widetilde{\mathcal{V}}_{f}}\left(v_{s}-v_{K}\right) \boldsymbol{g}_{f, s} \cdot\left(\boldsymbol{x}-\boldsymbol{x}_{\partial f}\right) \quad \nabla \pi_{f}^{\nabla} v=\sum_{s \in \mathcal{V}_{f} \cup \widetilde{\mathcal{V}}_{f}}\left(v_{s}-v_{K}\right) \boldsymbol{g}_{f, s}
$$

where

$$
\boldsymbol{x}_{\partial f}=\frac{1}{|\partial f|} \int_{\partial f} \boldsymbol{x}=\sum_{e \in \mathcal{E}_{f} \cup \widetilde{\mathcal{E}}_{f}} \frac{|e|}{|\partial f|} \boldsymbol{x}_{e} .
$$

As usual, we explicit the operator $\pi_{K}^{\nabla}$ for functions of $W(K)$. Using our definition of $W(K)$ and $W(f)$, we get that:

$$
\int_{\partial K} v \boldsymbol{n}_{\partial K}=\sum_{f \in \mathcal{S F}_{K}} \int_{f}\left(v-v_{K}\right) \boldsymbol{n}_{K, f}=\sum_{f \in \mathcal{S F}_{K}} \int_{f}\left(\pi_{f}^{\nabla}(v)-v_{K}\right) \boldsymbol{n}_{K, f} .
$$

This leads to:

$$
\nabla \pi_{K}^{\nabla} v=\sum_{f \in \mathcal{S} \mathcal{F}_{K}}\left(\sum_{s \in \mathcal{V}_{f} \cup \tilde{\mathcal{V}}_{f}} \frac{|f|}{|K|}\left(m_{f, s}+\boldsymbol{g}_{f, s} \cdot\left(\boldsymbol{x}_{f}-\boldsymbol{x}_{\partial f}\right)\right)\left(v_{s}-v_{K}\right) \boldsymbol{n}_{K, f}\right) .
$$

Then, recall that for any $s \in \widetilde{\mathcal{V}}_{f}$, if $f \in \mathcal{S F}_{\sigma}$, we have:

$$
v_{s}=\sum_{s^{\prime} \in \mathcal{V}_{\sigma}} \rho_{\sigma, s, s^{\prime}} v_{s^{\prime}} \quad \sum_{s^{\prime} \in \mathcal{V}_{\sigma}} \rho_{\sigma, s, s^{\prime}}=1,
$$


where we still denote $\rho_{\sigma, s, s^{\prime}}=\rho_{e, s, s^{\prime}}$ when $s \in \widetilde{\mathcal{V}}_{\partial \sigma}$ and $s \in e$ to simplify notations. Then:

$$
\begin{aligned}
\nabla \pi_{K}^{\nabla} v= & \sum_{f \in \mathcal{S} \mathcal{F}_{K}}\left(\sum_{s \in \mathcal{V}_{f}} \frac{|f|}{|K|}\left(m_{f, s}+\boldsymbol{g}_{f, s} \cdot\left(\boldsymbol{x}_{f}-\boldsymbol{x}_{\partial f}\right)\right)\left(v_{s}-v_{K}\right) \boldsymbol{n}_{K, f}\right. \\
& \left.+\sum_{s \in \tilde{\mathcal{V}}_{f}} \sum_{s^{\prime} \in \mathcal{V}_{\sigma}, f \in \mathcal{S} \mathcal{F}_{\sigma}} \frac{|f|}{|K|}\left(m_{f, s}+\boldsymbol{g}_{f, s} \cdot\left(\boldsymbol{x}_{f}-\boldsymbol{x}_{\partial f}\right)\right) \rho_{\sigma, s, s^{\prime}}\left(v_{s^{\prime}}-v_{K}\right) \boldsymbol{n}_{K, f}\right) .
\end{aligned}
$$

Denoting

$$
p_{f, s}=\left(m_{f, s}+\boldsymbol{g}_{f, s} \cdot\left(\boldsymbol{x}_{f}-\boldsymbol{x}_{\partial f}\right)\right)
$$

$\nabla \pi_{K}^{\nabla} v$ rewrites:

$$
\nabla \pi_{K}^{\nabla} v=\sum_{s \in \mathcal{V}_{K}} \sum_{f \in \mathcal{S} \mathcal{F}_{K} \cap \mathcal{S} \mathcal{F}_{s}} \frac{|f| p_{f, s}}{|K|}\left(v_{s}-v_{K}\right) \boldsymbol{n}_{K, f}+\sum_{s \in \mathcal{V}_{K}} \sum_{\sigma \in \mathcal{F}_{K} \cap \mathcal{F}_{s}} \sum_{f \in \mathcal{S} \mathcal{F}_{\sigma}} \sum_{s^{\prime} \in \widetilde{\mathcal{V}}_{f}} \frac{|f| p_{f, s^{\prime}} \rho_{\sigma, s^{\prime}, s}}{|K|}\left(v_{s}-v_{K}\right) \boldsymbol{n}_{K, f} .
$$

Introducing

$$
\boldsymbol{g}_{K, s}=\sum_{f \in \mathcal{S F}_{K} \cap \mathcal{S} \mathcal{F}_{s}} \frac{|f| p_{f, s}}{|K|} \boldsymbol{n}_{K, f}+\sum_{\sigma \in \mathcal{F}_{K} \cap \mathcal{F}_{s}} \sum_{f \in \mathcal{S F}_{\sigma}} \sum_{s^{\prime} \in \tilde{\mathcal{V}}_{f}} \frac{|f| p_{f, s^{\prime}} \rho_{\sigma, s^{\prime}, s}}{|K|} \boldsymbol{n}_{K, f}
$$

we obtain:

$$
\nabla \pi_{K}^{\nabla} v=\sum_{s \in \mathcal{V}_{K}}\left(v_{s}-v_{K}\right) \boldsymbol{g}_{K, s} .
$$

Exactly as in dimension 2, we can equivalently rewrite:

$$
\pi_{K}^{\nabla} v=v_{K}+\sum_{s \in \mathcal{V}_{K}}\left(v_{s}-v_{K}\right) \boldsymbol{g}_{K, s} \cdot\left(\boldsymbol{x}-\boldsymbol{x}_{K}\right) \quad \nabla \pi_{K}^{\nabla} v=\sum_{s \in \mathcal{V}_{K}}\left(v_{s}-v_{K}\right) \boldsymbol{g}_{K, s},
$$

but with a more complicated formula for the $\boldsymbol{g}_{K, s}$. As we have kept the same analytical form for our projectors, we can immediately deduce from the results in dimension 2 that the bilinear form $a(\cdot, \cdot)$ rewrites

$$
a_{h}(u, v)=\sum_{K \in \mathcal{T}} \sum_{s \in \mathcal{V}_{K}} F_{K, s}(u)\left(v_{K}-v_{s}\right),
$$

with the fluxes given by

$$
F_{K, s}(u)=\sum_{s^{\prime} \in \mathcal{V}_{K}} \mathbb{A}_{K}^{s^{\prime}, s}\left(u_{K}-u_{s^{\prime}}\right)
$$

and the matrix $\mathbb{A}_{K}$ given by

$$
\mid \begin{aligned}
& \mathbb{A}_{K}^{s, s^{\prime}}=|K| \Lambda_{K} \boldsymbol{g}_{K, s} \cdot \boldsymbol{g}_{K, s^{\prime}}+h_{K}^{d-2} \mathbb{S}_{K}^{s, s^{\prime}}, \\
& \mathbb{S}_{K}^{s, s^{\prime}}=y_{K,,^{\prime}}^{T} S_{K} y_{K, s}, \\
& y_{K, s}=\left(y_{K, s}^{s^{\prime \prime}}\right)_{s^{\prime \prime} \in \mathcal{V}_{K}}, \quad y_{K, s}^{s^{\prime \prime}}=\delta_{s, s^{\prime \prime}}-\boldsymbol{g}_{K, s} \cdot\left(\boldsymbol{x}_{s^{\prime \prime}}-\boldsymbol{x}_{K}\right) .
\end{aligned}
$$

Remark 3.7. As announced before, in dimension 3 the derivation of our fluxes $F_{K, s}$ is quite different from that of the original $3 \mathrm{~d}-\mathrm{VAG}$ scheme of [22], as it is based on the projectors $\pi_{f}^{\nabla}$ and not on barycentric interpolators. 
However there remains an interesting link between the two constructions. To make it clearer, notice that we can rewrite

$$
\boldsymbol{g}_{K, s}=\sum_{f \in \mathcal{S} \mathcal{F}_{K} \cap \mathcal{S} \mathcal{F}_{s}} \frac{|f| \beta_{f, s}}{|K|} \boldsymbol{n}_{K, f} \quad \text { with } \quad \beta_{f, s}=p_{f, s}+\sum_{s^{\prime} \in \tilde{\mathcal{V}}_{f}, f \in \mathcal{S} \mathcal{F}_{\sigma}} p_{f, s^{\prime}} \rho_{\sigma, s^{\prime}, s} .
$$

The question is now whether the $\beta_{f, s}$ are barycentric coefficients for the subface $f$ or not, i.e. do they satisfy

$$
\sum_{s \in \mathcal{V}_{\sigma}, f \in \mathcal{S} \mathcal{F}_{\sigma}} \beta_{f, s} \boldsymbol{x}_{s}=\boldsymbol{x}_{f} \quad \text { and } \sum_{s \in \mathcal{V}_{\sigma}, f \in \mathcal{S} \mathcal{F}_{\sigma}} \beta_{f, s}=1
$$

We have:

$$
\begin{gathered}
\sum_{s \in \mathcal{V}_{\sigma}, f \in \mathcal{S} \mathcal{F}_{\sigma}}\left(m_{f, s}+\sum_{s^{\prime} \in \widetilde{\mathcal{V}}_{f}, f \in \mathcal{S} \mathcal{F}_{\sigma}} m_{f, s^{\prime}} \rho_{\sigma, s^{\prime}, s}\right) \boldsymbol{x}_{s}= \\
\sum_{s \in \mathcal{V}_{\sigma}, f \in \mathcal{S} \mathcal{F}_{\sigma}}\left(\sum_{e \in\left(\mathcal{E}_{f} \cup \widetilde{\mathcal{E}}_{f}\right) \cap\left(\mathcal{E}_{s} \cup \widetilde{\mathcal{E}}_{s}\right)} \frac{|e|}{2|\partial f|} \boldsymbol{x}_{s}+\sum_{s^{\prime} \in \tilde{\mathcal{V}}_{f}, f \in \mathcal{S} \mathcal{F}_{\sigma}} \sum_{e \in\left(\mathcal{E}_{f} \cup \widetilde{\mathcal{E}}_{f}\right) \cap\left(\mathcal{E}_{s^{\prime}} \cup \widetilde{\mathcal{E}}_{s^{\prime}}\right)} \frac{|e|}{2|\partial f|} \rho_{\sigma, s^{\prime}, s} \boldsymbol{x}_{s}\right) \\
=\sum_{s \in \mathcal{V}_{f}} \sum_{e \in\left(\mathcal{E}_{f} \cup \widetilde{\mathcal{E}}_{f}\right) \cap\left(\mathcal{E}_{s} \cup \widetilde{\mathcal{E}}_{s}\right)} \frac{|e|}{2|\partial f|} \boldsymbol{x}_{s}+\sum_{s^{\prime} \in \widetilde{\mathcal{V}}_{f}} \sum_{e \in\left(\mathcal{E}_{f} \cup \widetilde{\mathcal{E}}_{f}\right) \cap\left(\mathcal{E}_{s^{\prime}} \cup \widetilde{\mathcal{E}}_{s^{\prime}}\right)} \frac{|e|}{2|\partial f|} \boldsymbol{x}_{s^{\prime}}=\sum_{e \in\left(\mathcal{E}_{f} \cup \widetilde{\mathcal{E}}_{f}\right)} \frac{|e|}{|\partial f|} \boldsymbol{x}_{e}=\boldsymbol{x}_{\partial_{f}} .
\end{gathered}
$$

Proceeding in the same way, we get:

$$
\begin{gathered}
\sum_{s \in \mathcal{V}_{\sigma}, f \in \mathcal{S} \mathcal{F}_{\sigma}}\left(\boldsymbol{g}_{f, s} \cdot\left(\boldsymbol{x}_{f}-\boldsymbol{x}_{\partial f}\right)+\sum_{s^{\prime} \in \widetilde{\mathcal{V}}_{f}, f \in \mathcal{S} \mathcal{F}_{\sigma}} \boldsymbol{g}_{f, s^{\prime}} \cdot\left(\boldsymbol{x}_{f}-\boldsymbol{x}_{\partial f}\right) \rho_{\sigma, s^{\prime}, s}\right) \boldsymbol{x}_{s} \\
=\sum_{s \in \mathcal{V}_{f} \in \in\left(\mathcal{E}_{f} \cup \widetilde{\mathcal{E}}_{f}\right) \cap\left(\mathcal{E}_{s} \cup \widetilde{\mathcal{E}}_{s}\right)} \frac{|e|}{2|f|} \boldsymbol{n}_{f, e} \cdot\left(\boldsymbol{x}_{f}-\boldsymbol{x}_{\partial f}\right) \boldsymbol{x}_{s}+\sum_{s^{\prime} \in \widetilde{\mathcal{V}}_{f}} \sum_{e \in\left(\mathcal{E}_{f} \cup \widetilde{\mathcal{E}}_{f}\right) \cap\left(\mathcal{E}_{s^{\prime}} \cup \widetilde{\mathcal{E}}_{s^{\prime}}\right)} \frac{|e|}{2|f|} \boldsymbol{n}_{f, e} \cdot\left(\boldsymbol{x}_{f}-\boldsymbol{x}_{\partial f}\right) \boldsymbol{x}_{s^{\prime}} \\
=\sum_{e \in\left(\mathcal{E}_{f} \cup \widetilde{\mathcal{E}}_{f}\right)} \frac{|e|}{|f|} \boldsymbol{n}_{f, e} \cdot\left(\boldsymbol{x}_{f}-\boldsymbol{x}_{\partial f}\right) \boldsymbol{x}_{e}=\boldsymbol{x}_{f}-\boldsymbol{x}_{\partial f}
\end{gathered}
$$

and thus the first part of (3.41) is satisfied. Then

$$
\sum_{s \in \mathcal{V}_{\sigma}, f \in \mathcal{S} \mathcal{F}_{\sigma}}\left(m_{f, s}+\sum_{s^{\prime} \in \tilde{\mathcal{V}}_{f}, f \in \mathcal{S} \mathcal{F}_{\sigma}} m_{f, s^{\prime}} \rho_{\sigma, s^{\prime}, s}\right)=\sum_{e \in\left(\mathcal{E}_{f} \cup \widetilde{\mathcal{E}}_{f}\right)} \frac{|e|}{|\partial f|}=1
$$

and

$$
\sum_{s \in \mathcal{V}_{\sigma}, f \in \mathcal{S} \mathcal{F}_{\sigma}}\left(\boldsymbol{g}_{f, s} \cdot\left(\boldsymbol{x}_{f}-\boldsymbol{x}_{\partial f}\right)+\sum_{s^{\prime} \in \tilde{\mathcal{V}}_{f}, f \in \mathcal{S} \mathcal{F}_{\sigma}} \boldsymbol{g}_{f, s^{\prime}} \cdot\left(\boldsymbol{x}_{f}-\boldsymbol{x}_{\partial f}\right) \rho_{\sigma, s^{\prime}, s}\right)=\sum_{e \in\left(\mathcal{E}_{f} \cup \widetilde{\mathcal{E}}_{f}\right)} \frac{|e|}{|f|} \boldsymbol{n}_{f, e} \cdot\left(\boldsymbol{x}_{f}-\boldsymbol{x}_{\partial f}\right)=0
$$

implies the second part. This means that our scheme could be considered as a very special VAG scheme with carefully designed barycentric weights. However the converse is very unlikely: not any barycentric interpolation formula allows a clear reinterpretation of the VAG scheme as a conforming variational approximation with well identified local spaces on each cell. Thus, our 3d fluxes seem to constitute a very interesting subfamily that allows a pleasant variational interpretation in $W(K)$ of the scheme, and in the process gives access to the wide 
range of admissible meshes of the mimetic technology (a classical consistency analysis without this variational framework would in particular be very difficult to conduct on non star-shaped cells). We insist on the fact that our scheme provides a conforming approximation, which a generic barycentric formula in $3 \mathrm{~d}$ cannot guarantee: this is a direct consequence of the VEM approach we followed to design the method. As in dimension 2, our scheme differs from the original VAG scheme through its stabilization part: a general framework following the lines of [18] that allows a matrix as stabilization parameter could again be introduced to unify both approaches, at least formally.

Finally, it is also clear that our construction is different from the alternative VAG construction described in $[8,23,24,28]$, which uses a piecewise linear reconstruction based on finite elements and a splitting of each cell into simplices, thus strongly requiring cells to be star-shaped.

\subsection{Reaction term}

We now turn to the approximation of the reaction term in $(2.1)$. We define, $\forall(u, v) \in W(K)$ :

$$
r_{h}(u, v)=\sum_{K \in \mathcal{T}} r_{h}^{K}(u, v) \quad \text { where } \quad r_{h}^{K}(u, v)=r^{K}\left(\pi_{0, K}(\beta) ; \pi_{0, K}(u), \pi_{0, K}(v)\right)+s_{0}^{K}\left(u-\pi_{0, K}(u), v-\pi_{0, K}(v)\right)
$$

where

$$
s_{0}^{K}(u, v)=h_{K}^{d} \sum_{s \in \mathcal{V}_{K}} \sum_{s^{\prime} \in \mathcal{V}_{K}} \mathbb{B}_{K}^{s, s^{\prime}} u\left(\boldsymbol{x}_{s}\right) v\left(\boldsymbol{x}_{s}\right)
$$

with $\mathbb{B}_{K}=\left(\mathbb{B}_{K}^{s, s^{\prime}}\right)_{s, s^{\prime} \in \mathcal{V}_{K}}$ any symmetric positive matrix independent on $h$. Denoting $\beta_{K}=\pi_{0, K}(\beta)$, we get for any $(u, v) \in H^{1}(K)^{2}$ :

$$
r_{h}^{K}(u, v)=\left(\pi_{0, K}(\beta) \pi_{0, K}(u), \pi_{0, K}(v)\right)_{0, K}+h_{K}^{d} \sum_{s \in \mathcal{V}_{K}} \sum_{s^{\prime} \in \mathcal{V}_{K}} \mathbb{B}_{K}^{s, s^{\prime}}\left(u_{s}-u_{K}\right)\left(v_{s^{\prime}}-v_{K}\right) .
$$

After a straightforward computation, this leads to:

$$
r_{h}^{K}(u, v)=|K| \beta_{K} u_{K} v_{K}+\sum_{s \in \mathcal{V}_{K}} F_{K, s}^{R}(u)\left(v_{K}-v_{s}\right)
$$

where the "reaction fluxes" are defined by:

$$
F_{K, s}^{R}(u)=h_{K}^{d} \sum_{s^{\prime} \in \mathcal{V}_{K}} \mathbb{B}_{K}^{s^{\prime}, s}\left(u_{K}-u_{s}^{\prime}\right) .
$$

Remark 3.8. The second term of (3.42), which gives birth to these surprising "reaction fluxes", is not needed to ensure convergence. However, as will be illustrated by numerical experiments, it greatly improves the behavior of the scheme when the diffusion tensor is vanishing. In [8,23,24], a different approach is proposed to ensure this additional robustness: an abstract volume decomposition of each cell $K \in \mathcal{T}$ is introduced, by assuming that one has a decomposition of the volume of $K$ under the form:

$$
K=K_{K} \bigcup_{s \in \mathcal{V}_{K}} K_{s}
$$

On each of these volumes, one approximates the unknown by the associated dof value, the corresponding approximation of the reaction term being thus given by:

$$
\sum_{K \in \mathcal{T}} \pi_{0, K}(\beta)\left(\left|K_{K}\right| u_{K} v_{K}+\sum_{s \in \mathcal{V}_{K}}\left|K_{s}\right| u_{s} v_{s}\right) .
$$


Doing so, we would give a volume to each vertex, but without modifying the fluxes between cell and vertices, contrary to our scheme that gives no volume to vertices but modify the fluxes.

Proceeding as in $[13,14]$, it is not difficult to establish that:

Lemma 3.9. There exists $b_{*} \geq 0$ and $b^{*}>0$, depending on $\rho$ but independent on $h$, such that for any $(u, v) \in$ $W(K)$

$$
r_{h}^{K}(u, v) \leq b^{*}\|u\|_{L^{2}(K)} \mid\|v\|_{L^{2}(K)}
$$

and for any $u \in W(K)$

$$
b_{*}\|u\|_{L^{2}(K)}^{2} \leq r_{h}^{K}(u, u) .
$$

For any $u \in W(K)$ and any $q \in \mathbb{P}_{0}(K)$,

$$
r_{h}^{K}(u, q)=\left(\pi_{0, K}(\beta) u, q\right)_{0, K} .
$$

\subsection{Finite volume formulation}

Combining all the previous definitions, we approximate our model problem (2.1) by the discrete variational formulation, with $W_{0}=W \cap H_{0}^{1}(\Omega)$ :

$$
\begin{aligned}
& \text { Find } u \in W_{0} \text { such that for all } v \in W_{0} \\
& \mathcal{A}_{h}(u, v)=a_{h}(u, v)+r_{h}(u, v)=\sum_{K \in \mathcal{T}} \int_{K} \pi_{0, K}(f) v .
\end{aligned}
$$

Using the flux formulation of each part, we get, denoting $f_{K}=\pi_{0, K}(f)$ :

$$
\sum_{K \in \mathcal{T}} \sum_{s \in \mathcal{V}_{K}}\left(F_{K, s}(u)+F_{K, s}^{R}(u)\right)\left(v_{K}-v_{s}\right)+\sum_{K \in \mathcal{T}}|K| \beta_{K} u_{K} v_{K}=\sum_{K \in \mathcal{T}}|K| f_{K} v_{K} .
$$

Taking one degree of freedom of $v$ equal to one and all others equal to 0 , and doing so for every dof, we obtain

$$
\mid \begin{array}{ll}
|K| \beta_{K} u_{K}+\sum_{s \in \mathcal{V}_{K}} F_{K, s}(u)+F_{k, s}^{R}(u)=|K| f_{K} & \forall K \in \mathcal{T} \\
\sum_{K \in \mathcal{T}_{s}} F_{s, K}(u)+F_{s, K}^{R}(u)=0 & \forall s \in \mathcal{V}_{i n t} \\
u_{s}=0 & \forall s \in \mathcal{V}_{\text {ext }}
\end{array}
$$

where we have denoted

$$
F_{s, K}(u)=-F_{K, s}(u)=\sum_{s^{\prime} \in \mathcal{V}_{K}} \mathbb{A}_{K}^{s^{\prime}, s}\left(u_{s^{\prime}}-u_{K}\right) \quad \forall u \in W(K)
$$

and

$$
F_{s, K}^{R}(u)=-F_{K, s}^{R}(u)=\sum_{s^{\prime} \in \mathcal{V}_{K}} \mathbb{B}_{K}^{s^{\prime}, s}\left(u_{s^{\prime}}-u_{K}\right) \quad \forall u \in W(K) .
$$

In other words, our method shares the same analytical formulation than classical finite volume methods, with some "real" volumes (those associated with cells) exchanging with some "virtual" ones (those associated with vertices, as vertices are entities with no volume). We also recover the balance of the global diffusion-reaction fluxes ensured by (3.50) and (3.51). This is due to the introduction of our additional cell degree of freedom, as well as the careful choice of stabilization terms. Notice that we were able to obtain explicit expressions for each 
quantity involved in our scheme, which greatly simplifies its implementation: no local inversion, such as those required for MPFA schemes, is necessary to compute the fluxes. Also notice that we can use static condensation to drastically decrease the size of the linear system: the cell equations in (3.49) can be locally inverted, thus reducing the linear system to the vertex unknowns only.

Let us mention that it seemed logical to call our new method a "virtual volume" method, as we obtain a finite volume scheme derived from virtual element principles and its associated virtual basis, and because of the formal exchange between real and virtual volumes.

\section{Convergence analysis}

In this section, we consider the convergence properties of our virtual volume method. The variational approach we followed, based on the mimetic technology, will prove all its interest here: indeed, most of the work will be greatly simplified, as we will be able to use directly classical approximation properties. This section follows exactly the lines of $[7,14]$ applied to our enhanced VEM space, as the VEM technology provides convergence in a rather automatic way. Consequently, we claim no originality here: we only detail the convergence analysis for the sake of completeness and the reader's convenience.

A direct consequence of the optimal polynomial approximation properties of our mesh sequence is the following approximation result for the operator $\pi_{K}^{\nabla}$ :

Lemma 4.1. Let $d=2$ or 3 and $\mathcal{M}$ a mesh that has optimal polynomial approximation properties. Then, for any $u \in H^{2}(K)$

$$
\| \nabla u-\left.\nabla \pi_{K}^{\nabla}(u)\right|_{L^{2}(K)} \leq C_{\text {poly }} h_{K}|u|_{H^{2}(K)}
$$

Proof. By definition of $\pi_{K}^{\nabla}(u)$ and as for any $q \in \mathbb{P}_{1}(K), \nabla \pi_{K}^{\nabla}(q)=\nabla q$, we get:

$$
\left(\nabla u-\nabla \pi_{K}^{\nabla}(u), \nabla u-\nabla \pi_{K}^{\nabla}(u)\right)_{0, K}=\left(\nabla u-\nabla \pi_{K}^{\nabla}(u), \nabla u-\nabla q\right)_{0, K}
$$

which leads to

$$
\left\|\nabla u-\nabla \pi_{K}^{\nabla}(u)\right\|_{L^{2}(K)} \leq \inf _{q \in \mathbb{P}_{1}(K)}\|\nabla u-\nabla q\|_{L^{2}(K)}
$$

and concludes the proof.

Moreover, following Scott-Dupond theory, as a consequence of our regularity hypothesis (A1) on the mesh and as $\mathbb{P}_{1}(K) \subset W(K)$ it is not difficult to see that we have (see $[7,9,14]$ ):

Proposition 4.2. Let $d=2$ or 3 and $\mathcal{M}$ be a mesh of $\Omega$, that satisfies (A1). There exists $C_{W}>0$ such that for any $v \in H^{2}(K)$ there exists $w \in W$ such that for any $K \in \mathcal{T}$

$$
\| v-\left.w\right|_{L^{2}(K)}+h_{K}|v-w|_{H^{1}(K)} \leq C_{W} h_{K}^{2}|v|_{H^{2}(K)} .
$$

\subsection{Convergence theorems and error estimates}

Convergence results are based on the following abstract result:

Proposition 4.3. Let $d=2$ or $3, \mathcal{M}$ be a mesh of $\Omega$ that satisfies (A1), and let $u$ be the unique variational solution of (2.1). Then, there exists $C>0$ depending on $\rho$ but independent on $h$ such that the solution $u_{h}$ of (3.48) satisfies, for any $u_{I} \in W$, any $u_{\pi, 1}$ piecewise in $\mathbb{P}_{1}(K)$ for any $K \in \mathcal{T}$, and any $u_{\pi, 0}$ piecewise in $\mathbb{P}_{0}(K)$ for any $K \in \mathcal{T}$ :

$$
\begin{aligned}
\left\|u-u_{h}\right\|_{H^{1}(\Omega)} \leq & C\left(\left\|f-\pi_{\mathcal{T}, 0}(f)\right\|_{L^{2}(\Omega)}+\left|u-u_{I}\right|_{H^{1}(\Omega)}+\left|u-u_{\pi, 1}\right|_{1, h}+\left\|u-u_{\pi, 0}\right\|_{L^{2}(\Omega)}+\left\|u-u_{I}\right\|_{L^{2}(\Omega)}\right. \\
& \left.+\sup _{K \in \mathcal{T}}\left\|\Lambda-\Lambda_{K}||_{L^{\infty}(K)}|u|_{H^{1}(\Omega)}+\sup _{K \in \mathcal{T}}\right\| \beta-\beta_{K}\left\|_{L^{\infty}(K)}\right\| u \|_{L^{2}(\Omega)}\right)
\end{aligned}
$$


where $\Lambda_{K}=\pi_{0, K}(\Lambda), \beta_{K}=\pi_{0, K}(\beta)$ and:

$$
|u|_{1, h}^{2}=\sum_{K \in \mathcal{T}}\|\nabla u\|_{L^{2}(K)}^{2}
$$

and $\pi_{\mathcal{T}, 0}(f)=\pi_{0, K}(f)$ in $K, \forall K \in \mathcal{T}$.

Proof. Let $u$ be in $H^{1}, u_{I}$ in $W, u_{\pi, 1}$ be piecewise in $\mathbb{P}_{1}(K)$ and $u_{\pi, 0}$ be piecewise in $\mathbb{P}_{0}(K)$. Then, using the stability of the diffusion and reaction part on each $K$, the hypothesis on $\Lambda$ and $\beta$, we deduce that there exist a constant $\alpha>0$ and a constant $\eta \geq 0$, both independent on $h$, such that, setting $\delta_{h}=u_{h}-u_{I}$ :

$$
\begin{gathered}
\eta\left\|\delta_{h}\right\|_{L^{2}(\Omega)}^{2}+\alpha\left|\delta_{h}\right|_{H^{1}(\Omega)}^{2} \leq \mathcal{A}_{h}\left(\delta_{h}, \delta_{h}\right)=\sum_{K \in \mathcal{T}} a_{h}^{K}\left(u_{h}, \delta_{h}\right)+r_{h}^{K}\left(u_{h}, \delta_{h}\right)-a_{h}^{K}\left(u_{I}, \delta_{h}\right)-r_{h}^{K}\left(u_{I}, \delta_{h}\right) \\
=\sum_{K \in \mathcal{T}}\left(f_{K}, \delta_{h}\right)_{0, K}-a_{h}^{K}\left(u_{I}, \delta_{h}\right)-r_{h}^{K}\left(u_{I}, \delta_{h}\right) \text { (using (3.48)) } \\
\left.=\sum_{K \in \mathcal{T}}\left(f_{K}, \delta_{h}\right)_{0, K}-a_{h}^{K}\left(u_{I}-u_{\pi, 1}, \delta_{h}\right)-a_{h}^{K}\left(u_{\pi, 1}, \delta_{h}\right)-r_{h}^{K}\left(u_{I}-u_{\pi, 0}, \delta_{h}\right)-r_{h}^{K}\left(u_{\pi, 0}, \delta_{h}\right) \text { (adding } \pm u_{\pi, 1} \text { and } \pm u_{\pi, 0}\right) \\
=\sum_{K \in \mathcal{T}}\left(f_{K}, \delta_{h}\right)_{0, K}-a_{h}^{K}\left(u_{I}-u_{\pi, 1}, \delta_{h}\right)-r_{h}^{K}\left(u_{I}-u_{\pi, 0}, \delta_{h}\right)-a^{K}\left(\Lambda_{K} ; u_{\pi, 1}, \delta_{h}\right)-r^{K}\left(u_{\pi, 0}, \delta_{h}\right)
\end{gathered}
$$

(using (3.12),(3.32) and (3.46)). Then, adding $\pm u$ two times, we get

$$
\begin{aligned}
\eta\left\|\delta_{h}\right\|_{L^{2}(\Omega)}^{2}+\alpha\left|\delta_{h}\right|_{H^{1}(\Omega)}^{2} \leq & \sum_{K \in \mathcal{T}}\left(f_{K}, \delta_{h}\right)_{0, K}-a_{h}^{K}\left(u_{I}-u_{\pi, 1}, \delta_{h}\right)-r_{h}^{K}\left(u_{I}-u_{\pi, 0}, \delta_{h}\right)-a^{K}\left(\Lambda_{K} ; u_{\pi, 1}-u, \delta_{h}\right) \\
& -a^{K}\left(\Lambda_{K} ; u, \delta_{h}\right)-r^{K}\left(\beta_{K} ; u_{\pi, 0}-u, \delta_{h}\right)-r^{K}\left(\beta_{K} ; u, \delta_{h}\right),
\end{aligned}
$$

which leads to, using (2.1)

$$
\begin{gathered}
\eta\left\|\delta_{h}\right\|_{L^{2}(\Omega)}^{2}+\alpha\left|\delta_{h}\right|_{H^{1}(\Omega)}^{2} \leq \sum_{K \in \mathcal{T}}\left(\pi_{0, K}(f)-f, \delta_{h}\right)_{0, K}-a_{h}^{K}\left(u_{I}-u_{\pi, 1}, \delta_{h}\right)-r_{h}^{K}\left(u_{I}-u_{\pi, 0}, \delta_{h}\right) \\
-a^{K}\left(\Lambda_{K} ; u_{\pi, 1}-u, \delta_{h}\right)-a^{K}\left(\Lambda_{K}-\Lambda ; u, \delta_{h}\right)-r^{K}\left(\beta_{K} ; u_{\pi, 0}-u, \delta_{h}\right)-r^{K}\left(\beta_{K}-\beta ; u, \delta_{h}\right) .
\end{gathered}
$$

Using the definition of $a^{K}$ and $r^{K}$ and the continuity bounds on $a_{h}^{K}$ and $r_{h}^{K}$, we easily obtain the existence of some $C>0$ independent on $h$ such that:

$$
\begin{aligned}
& \eta\left\|\delta_{h}\right\|_{L^{2}(\Omega)}^{2}+\alpha\left|\delta_{h}\right|_{H^{1}(\Omega)}^{2} \leq C\left(\left\|f-\left.\pi_{\mathcal{T}, 0}(f)\right|_{L^{2}(\Omega)}+\left|u_{I}-u_{\pi, 1}\right|_{1, h}+\left|u-u_{\pi, 1}\right|_{1, h}+\right\| u_{I}-u_{\pi, 0} \|_{L^{2}(\Omega)}\right. \\
& \left.\quad+\left\|u-u_{\pi, 0}||_{L^{2}(\Omega)}+\sup _{K \in \mathcal{T}}\right\| \Lambda-\pi_{0, K}(\Lambda)\left\|_{L^{\infty}(K)}|u|_{H^{1}(\Omega)}+\sup _{K \in \mathcal{T}}\right\| \beta-\left.\pi_{0, K}(\beta)\left\|_{L^{\infty}(K)}\right\| u\right|_{L^{2}(\Omega)}\right) .
\end{aligned}
$$

and the result follows using the triangle inequality and Poincaré's inequality on $\Omega$.

As the mesh has optimal polynomial approximation properties, assuming that $f \in H^{1}(\Omega), \beta \in W^{1, \infty}(\Omega)$ and $\Lambda \in W^{1, \infty}\left(\Omega, \mathbb{M}_{3}(\mathbb{R})\right)$, there exists $C_{f}>0, C_{\beta}>0$ and $C_{\Lambda}>0$, all independent on $h$, such that for all $K \in \mathcal{T}_{h}$

$$
\left\|f-\pi_{0, K}(f)\right\|_{L^{2}(K)} \leq C_{f} h_{K} \quad\left\|\beta-\pi_{0, K}(\beta)\right\|_{L^{\infty}(K)} \leq C_{\beta} h_{K} \quad\left\|\Lambda-\pi_{0, K}(\Lambda)\right\|_{L^{\infty}(K)} \leq C_{\Lambda} h_{K} .
$$


Then, one immediately deduces:

Proposition 4.4. Let $d=2$ or 3 , and $\mathcal{M}$ be a mesh of $\Omega$, that satisfies (A1). Assume that the unique variational solution $u$ of (2.1) belongs to $H^{2}(\Omega)$, that $f \in H^{1}(\Omega), \beta \in W^{1, \infty}(\Omega)$ and $\Lambda \in W^{1, \infty}\left(\Omega, \mathbb{M}_{3}(\mathbb{R})\right)$. Then, there exists $C>0$ depending on $\rho$ but independent on $h$ such that the solution $u_{h}$ of (3.48) satisfies

$$
\left\|u-u_{h}\right\|_{L^{2}(\Omega)} \leq C h \quad \text { and } \quad\left\|\nabla u-\nabla \pi_{K}^{\nabla}\left(u_{h}\right)\right\|_{L^{2}(K)} \leq C h
$$

Using the usual duality argument, one could then establish error estimates in the $L^{2}(\Omega)$ norm assuming elliptic regularity (see [7] for details):

Proposition 4.5. We say that an open set $\Omega$ has elliptic regularity if and only if for all $g \in L^{2}(\Omega)$, the unique solution $w \in H_{0}^{1}(\Omega)$ of

$$
-\operatorname{div}(\Lambda \nabla w)+\beta w=g \quad \text { in } \Omega
$$

satisfies

$$
\|w\|_{H^{2}(\Omega)} \leq C_{e}\|g\|_{L^{2}(\Omega)}
$$

with $C_{e}>0$ only depending on $\Omega$.

Assume that $\Omega$ has elliptic regularity, and let $d=2$ or 3 , and $\mathcal{M}$ be a mesh of $\Omega$, that satisfies (A1). Assume that $f \in H^{1}(\Omega), \beta \in W^{1, \infty}(\Omega), \Lambda \in W^{1, \infty}\left(\Omega, \mathbb{M}_{3}(\mathbb{R})\right)$. Then, there exists $C>0$ depending on $\rho$ but independent on $h$ such that the solution $u_{h}$ of (3.48) satisfies

$$
\left\|u-u_{h}\right\|_{L^{2}(\Omega)} \leq C h^{2}
$$

We see that having a variational formulation on the space $W_{0}$ greatly simplifies the analysis. Also notice the apparent paradox inherent to our method: in principle, as we are trying to match the finite volume philosophy, one could expect that we perform an external approximation of the space $H_{0}^{1}(\Omega)$, to incorporate cellwise constant functions. However we used a discrete variational formulation on the space $W_{0}$, which is a subspace of $H_{0}^{1}(\Omega)$, thus the approximation is internal. The trick here is the VEM notion of consistency: being much weaker than the classical finite element one, as we are only imposing local consistency when tested against polynomials, it allows the stabilization part to not be an approximation of the diffusion matrix. Then well chosen stabilizations that preserve the flux balanced formulation as well as the choice for the second member are the key to recover local conservativity.

\section{NumERICAL EXPLORATION}

Before describing our tests cases, let us precise the exact form of the stabilization terms we use in practice. After some numerical experiments we set

$$
S_{K, s, s^{\prime}}=\delta_{s, s^{\prime}} \sum_{f \in \mathcal{S} \mathcal{F}_{K} \cap \mathcal{S} \mathcal{F}_{s}} \frac{|f| \Lambda_{K} \boldsymbol{n}_{K, f} \cdot \boldsymbol{n}_{K, f}}{\sum_{f^{\prime} \in \mathcal{S} \mathcal{F}_{K} \cap \mathcal{S} \mathcal{F}_{s}}\left|f^{\prime}\right|}
$$

and $\mathbb{B}_{K}^{s, s^{\prime}}=\gamma \beta_{K} \delta_{s, s^{\prime}}$, with $\gamma>0$ a real parameter allowing to control the diffusion added by the reaction fluxes on coarse meshes. The idea behind the choice (5.1) is simply to match the variations of the diffusion tensor, which roughly speaking makes the stability and coercivity constants much less dependent on the variations of $\Lambda$. Clearly, other choices can lead to more or less precise results, depending on the test case, and the above proposition is most certainly suboptimal. However, we felt that it gives sufficiently precise results to assess the potential of the method. Then, let us mention that for simplicity, the $L^{2}$ convergence results that will be plotted correspond to

$$
\sqrt{\sum_{K \in \mathcal{T}}|K|\left(u\left(\boldsymbol{x}_{K}\right)-u_{K}\right)^{2}}
$$


where $u$ is the exact solution. For any function in $H^{1+\alpha}$, this constitutes an order $1+\alpha$ approximation of the true $L^{2}$ error. For a function with such regularity, the best approximation error than could be hoped is of order $\min (2,2 \alpha)$, thus this approximation is sufficient to study convergence properties. Notice that the results developed in this paper can be extended to more general boundary conditions in a very straightforward and very usual manner: this explains why we will exhibit examples with in particular inhomogeneous Dirichlet boundary conditions.

For each convergence test, we will compare our virtual volume method (VVM) to the original Hybrid scheme (described for instance in [21]), to the original VAG scheme of [22] as well as the naturally stable variant VAGRT based on a tetrahedral subdivision of cells of [28] (like the original hybrid scheme, it strongly requires cells to be star-shaped). They serve as reference finite volume methods, as they share the properties of symmetry and coercivity with the VVM. The VVM, VAG and VAGR schemes have exactly the same number of unknowns (one per cell and vertex), while the Hybrid scheme has in general much more unknowns (one per cell and face). The constant in the stabilization term is taken equal to $d$ for the hybrid scheme, while it is taken equal to $\operatorname{car} d\left(\mathcal{V}_{K}\right)$ in each cell for the VAG scheme. Numerical experiments on the series of test cases we are going to show have proven these constants to be experimentally optimal. We also include the first order virtual element method in the comparison to assess the effect of the additional cell value on the precision of the results, although this last method does not offer a finite volume flux balanced formulation.

\subsection{Numerical results in dimension 2}

We begin our numerical exploration by considering the case of pure diffusion $(\beta=0)$. To assess the behavior of the method in presence of heterogeneity, we consider the three tests cases of $[4,25]$ : the domain $\Omega=] 0,1[\times] 0,1[$ is subdivided into four areas, denoted $D_{i}, i=1 . .4$, and we use a sequence of meshes fitting this partition (see Fig. 2).

For the first test case (named ConvTest1, see Fig. 3), we choose $\Lambda_{\left.\right|_{D_{1}}}=\lambda_{1} I d$ and $\Lambda_{\left.\right|_{\Omega \backslash D_{1}}}=\lambda_{2} I d$. A solution of $-\operatorname{div}(\Lambda \nabla u)=0$ is then given by:

$$
u=\mid \begin{array}{ll}
r^{\alpha} \cos (\alpha(\theta-\pi / 3)) & \text { for } \theta \in[0,2 \pi / 3] \\
\beta r^{\alpha} \cos (\alpha(4 \pi / 3-\theta)) & \text { for } \theta \in[2 \pi / 3,2 \pi]
\end{array}
$$

with $\alpha=(3 / \pi) \arctan \sqrt{1+2 / \kappa}$ and $\beta=\cos (\alpha \pi / 3) / \cos (2 \alpha \pi / 3)$, and $\kappa=\lambda_{1} / \lambda_{2}=0.1$. With the same tensor, another solution, corresponding to the second test case (named ConvTest2, see Fig. 3), is given by

$$
u=\mid \begin{array}{ll}
r^{\alpha} \sin (\alpha(\theta-\pi / 3)) & \text { for } \theta \in[0,2 \pi / 3] \\
\beta r^{\alpha} \sin (\alpha(4 \pi / 3-\theta)) & \text { for } \theta \in[2 \pi / 3,2 \pi]
\end{array}
$$
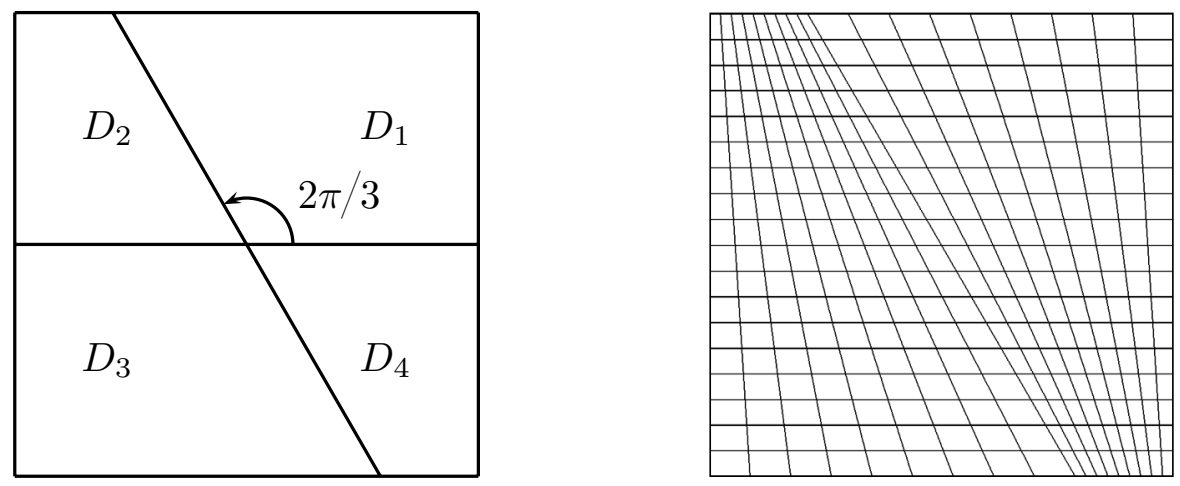

Figure 2. Domain subdivision and example of mesh. 

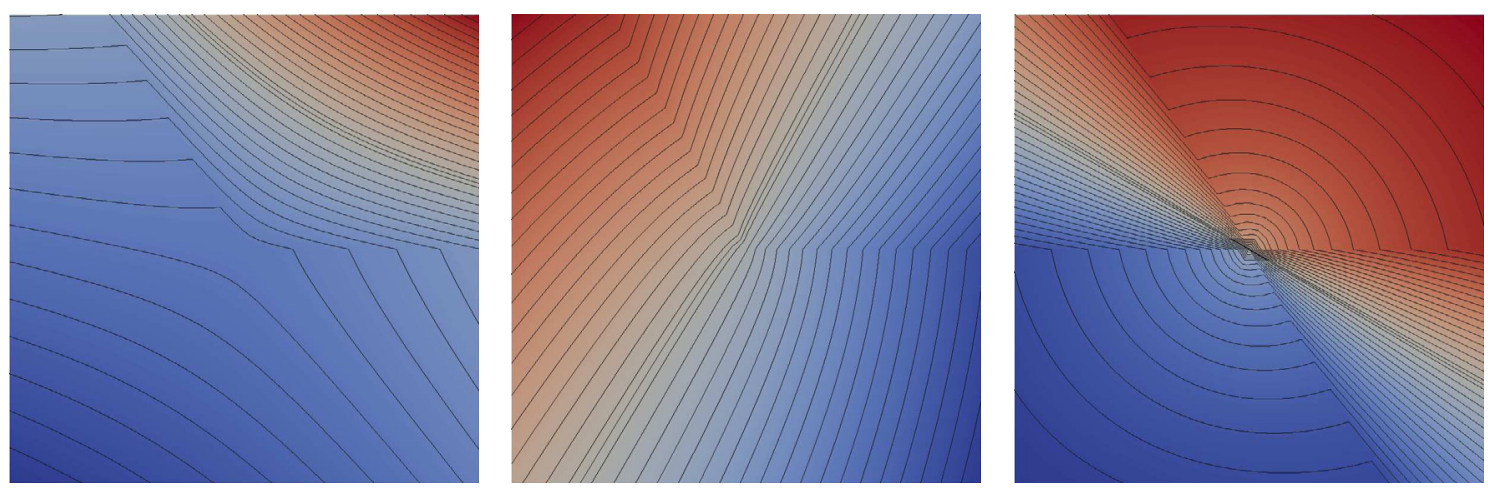

Figure 3. Isolines of solutions for ConvTest1, ConvTest2 and ConvTest3.
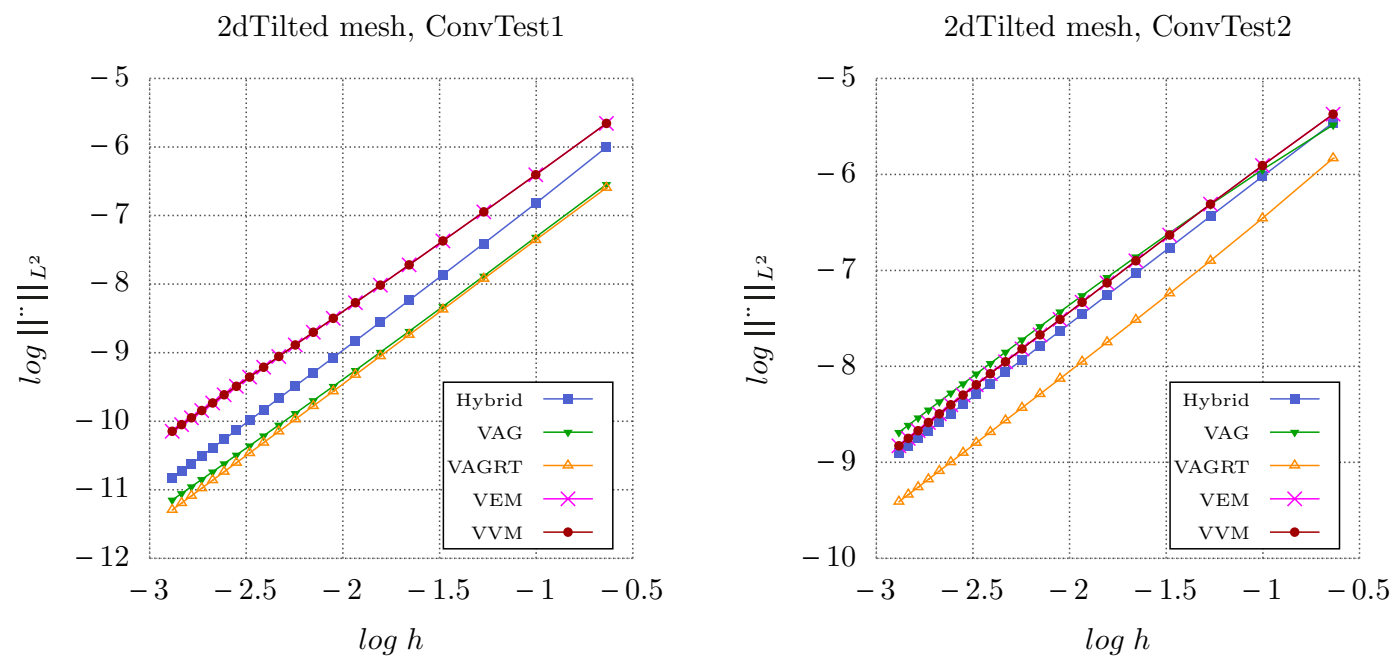

Figure 4. Convergence curves for ConvTest1 and ConvTest2.

with $\alpha=(3 / \pi) \arctan \sqrt{1+2 \kappa}$ and $\beta=1 /(2 \cos (\alpha \pi / 3))$, and $\kappa=\lambda_{1} / \lambda_{2}$.

In the case where $\Lambda_{\left.\right|_{D_{1}}}=\Lambda_{\left.\right|_{D_{3}}}=\lambda_{1} I d$ and $\Lambda_{\left.\right|_{D_{2}}}=\Lambda_{\left.\right|_{D_{4}}}=\lambda_{2} I d$, a solution with a stronger singularity at the origin, corresponding to the third test case (named ConvTest3, see Fig. 3), satisfies $u(r, \theta)=-u(r, \theta-\pi)$ and

$$
u=\mid \begin{array}{ll}
r^{\alpha} \cos (\alpha(\theta-\pi / 3)) & \text { for } \theta \in[0,2 \pi / 3] \\
\beta r^{\alpha} \sin (\alpha(5 \pi / 6-\theta)) & \text { for } \theta \in[2 \pi / 3, \pi]
\end{array}
$$

with $\alpha=(6 / \pi) \arctan (1 / \sqrt{1+2 \kappa})$ and $\beta=\cos (\alpha \pi / 3) / \sin (\alpha \pi / 6)$, and $\kappa=\lambda_{1} / \lambda_{2}=30$.

In each case, the solution belongs to $H^{1+\alpha-\epsilon}$ for any $\epsilon>0$. This means that the solutions of convTest1, ConvTest2 and ConvTest 3 have the respective approximate regularities $H^{2.29}, H^{1.79}$ and $H^{1.24}$, as $\alpha \approx 1.29$ for ConvTest $1, \alpha \approx 0.79$ for ConvTest2 and $\alpha \approx 0.24$ for ConvTest3. From the results displayed in Figures 4 and 5 , we deduce the approximate convergence orders of Table 1, which are in agreement with the optimal order of $\min (2,2 \alpha)$ which is 2 for the ConvTest1, 1.58 for ConvTest2 and 0.48 for ConvTest3. Notice that the three methods have a very close convergence behavior on these test cases. Then, to assess the behavior of the method 


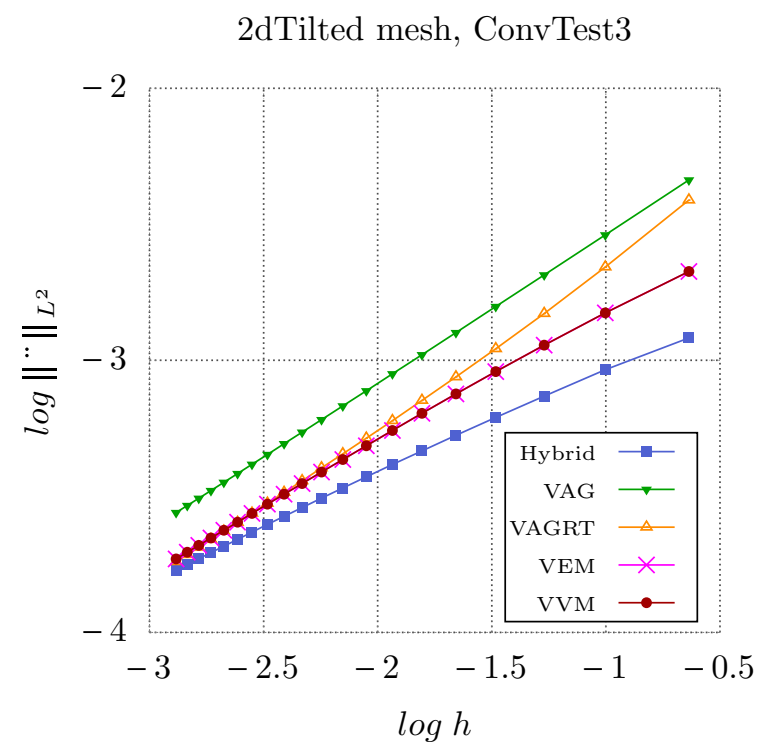

Figure 5. Convergence curves for ConvTest3.

TABle 1. Approximate orders of convergence for ConvTest1, ConvTest2 and ConvTest3.

\begin{tabular}{cccc}
\hline & ConvTest1 & ConvTest2 & ConvTest3 \\
\hline Hybrid & 2.12 & 1.52 & 0.40 \\
VAG & 2.01 & 1.49 & 0.54 \\
VAGRT & 2.08 & 1.54 & 0.55 \\
VEM & 1.98 & 1.57 & 0.49 \\
VVM & 1.98 & 1.57 & 0.49 \\
\hline
\end{tabular}

in presence of anisotropy and on distorted mesh, still on $\Omega=] 0,1[\times] 0,1[$, we consider

$$
u(x, y)=\sin (\pi x) \sin (\pi y) \quad \Lambda=\left(\begin{array}{ll}
1 & 0 \\
0 & \epsilon
\end{array}\right)
$$

with $\epsilon=10^{-3}$, which will be named ConvTest4. We consider five types of mesh sequences. The first one (2dDualDelaunay) is obtained by considering the dual meshes (the cell formed by joining the centers of the cell of the primal mesh) of a sequence of Delaunay meshes, while the second one (2dVoronoi) is obtained by considering the Voronoi meshes associated to the same sequence of Delaunay meshes. These two approaches generate polygonal meshes with quite generic cells. The third sequence (2dKershawBox) of meshes is a sequence of Kershaw meshes of the unit square, while the fourth one (2dCheckerBoardBox) is a sequence of checkerboard meshes of the unit square. These two sequences have only quadrangular cells which are distorted for the sequence 2dKershawBox, while the sequence 2dCheckerBoardBox allows to test the behavior of the method in presence of non conformities. The fifth sequence, named $2 d T I E$, illustrates how general meshes can be. From the results displayed in Figures 9-11, we deduce the approximate order of convergence of Table 2 by performing a linear regression only on the last points of the curves, to avoid the somewhat erratic behavior of some of the mesh sequences when $h$ is large and get significant results. In this case where the solution is smooth enough, the virtual volume method reaches the optimal order 2 on any mesh sequence. The hybrid scheme (in the original version of [21]) and the VAGRT scheme fail to converge on the 2dTIE mesh sequence, as cells are non star-shaped. Of course, using the generic stabilizations terms of the HMM generalization (see [18]) of the Hybrid scheme, 

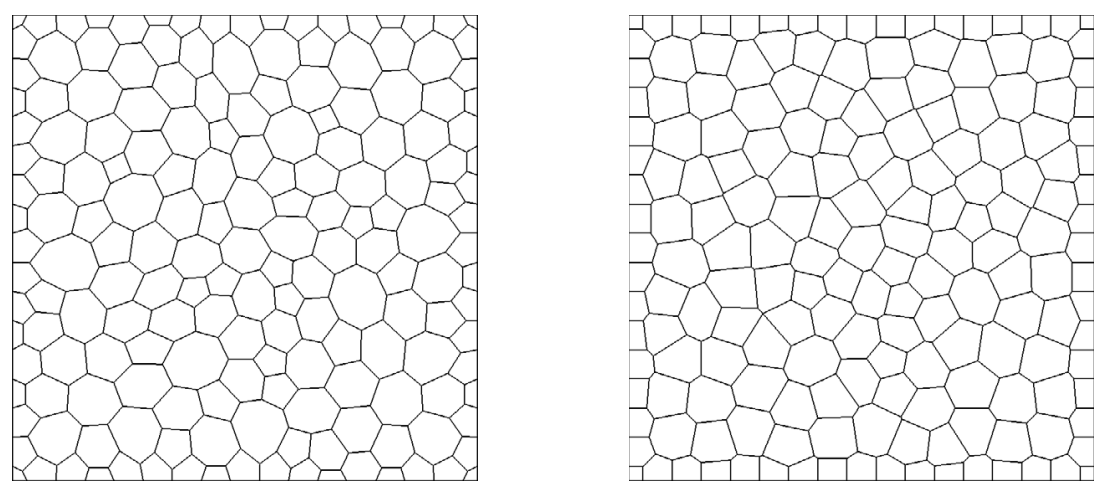

Figure 6. Example of meshes for the 2dDualDelaunay and 2dVoronoi mesh sequences.
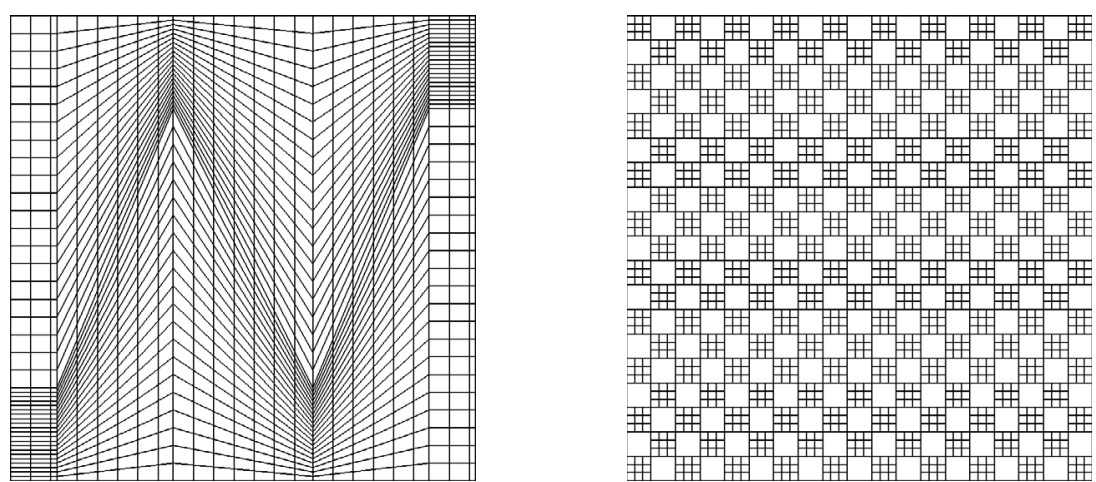

Figure 7. Example of meshes for the 2dKershawBox and 2dCheckerBoardBox mesh sequences.

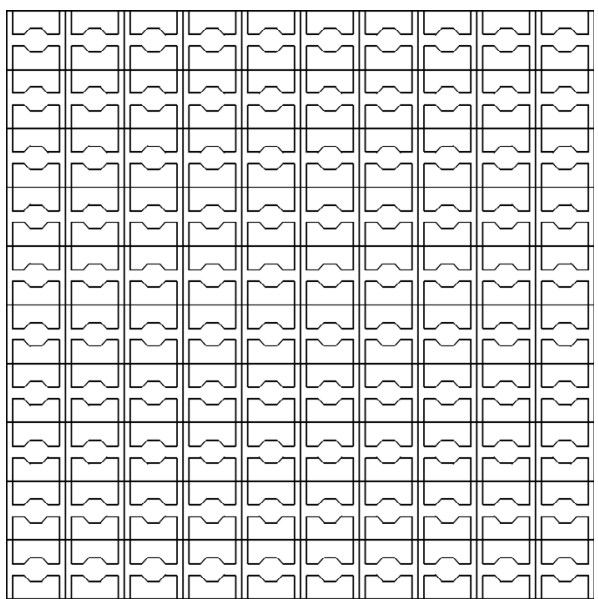

Figure 8. Example of meshes for the 2dTIE mesh sequence. 
TABle 2. Approximate orders of convergence for ConvTest4.

\begin{tabular}{lccccc}
\hline & 2dDualDelaunay & 2dVoronoi & 2dKershawBox & 2dCheckerBoardBox & 2dTIE \\
\hline Hybrid & 1.98 & 1.84 & 1.99 & 2.68 & - \\
VAG & 2.08 & 2.11 & 1.81 & 2.00 & 1.97 \\
VAGRT & 2.02 & 1.88 & 1.81 & 1.89 & - \\
VEM & 1.99 & 1.96 & 1.89 & 2.01 & 1.97 \\
VVM & 2.01 & 2.20 & 1.94 & 2.03 & 1.99 \\
\hline
\end{tabular}

2dDualDelaunay mesh, ConvTest4

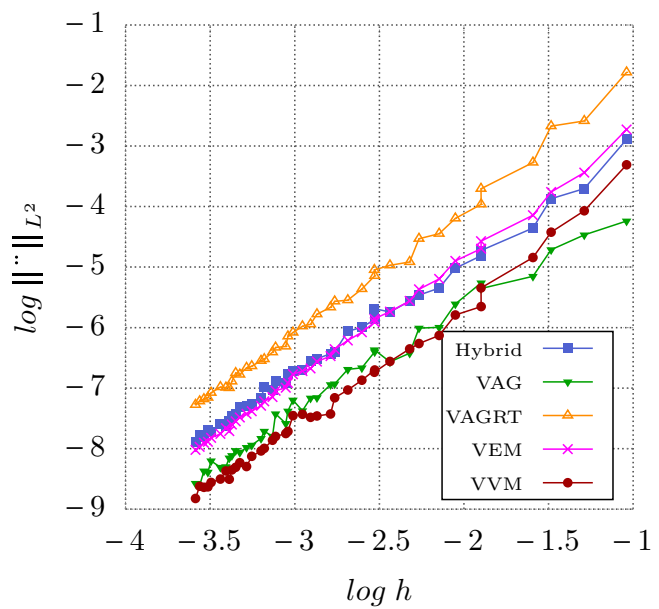

2dVoronoi mesh, ConvTest4

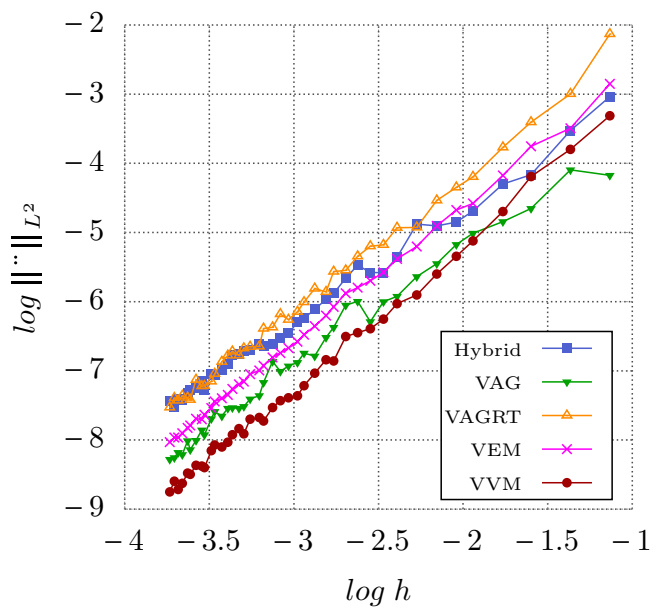

Figure 9. Convergence curves for ConvTest4 for the 2dDualDelaunay and 2dVoronoi mesh sequences.

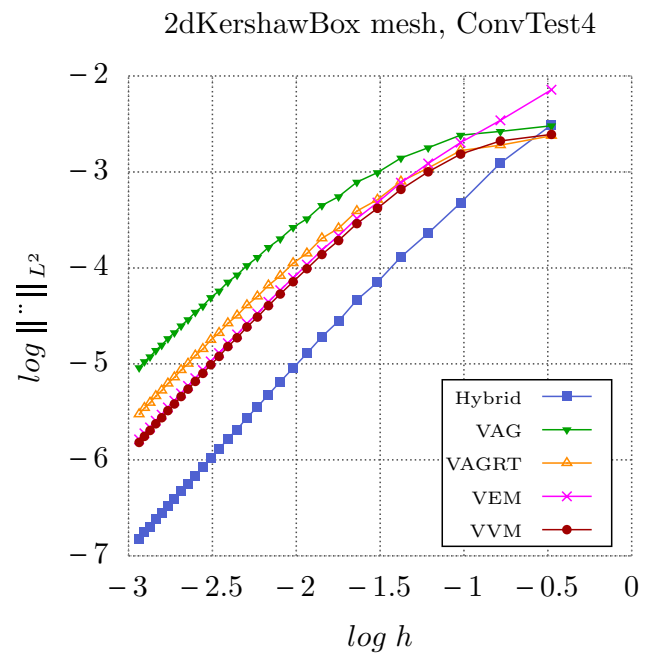

2dCheckerBoardBox mesh, ConvTest4

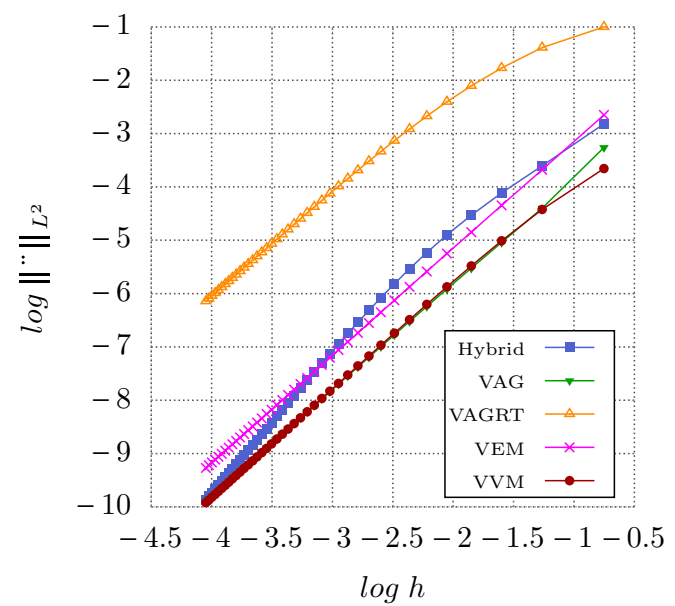

Figure 10. Convergence curves for ConvTest4 on the 2dKershawBox and 2dCheckerBoardBox mesh sequences. 


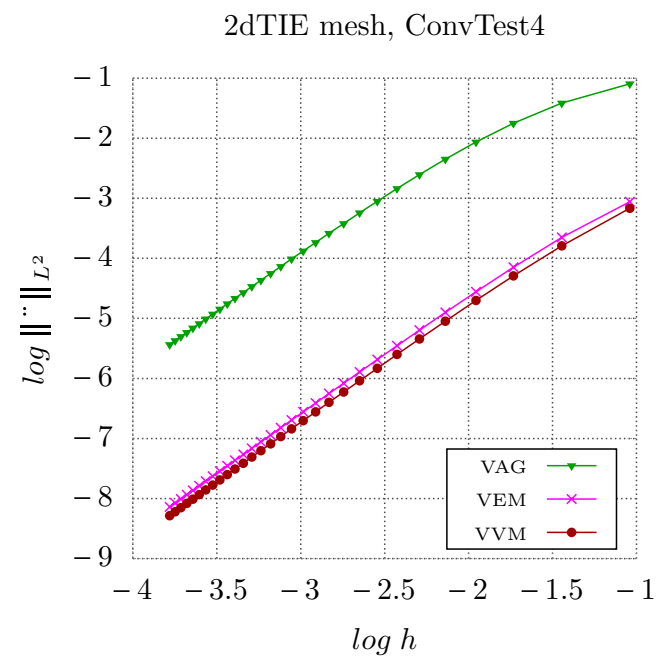

Figure 11. Convergence curves ConvTest4 on the 2dTIEMesh mesh sequence.
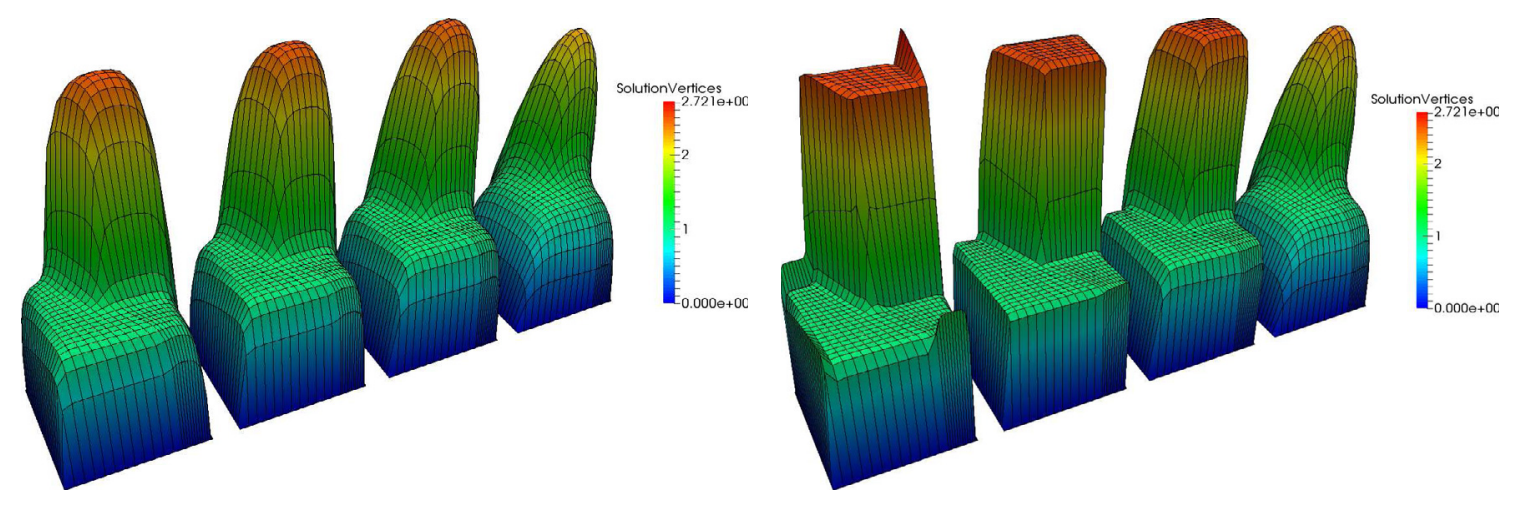

FiguRE 12. Extruded solutions for the reaction dominated case, $\gamma$ going from 0 on the left to 1 on the right in each figure, left: $\epsilon=10^{-3}$, right: $\epsilon=10^{-5}$.

convergence is retrieved. The virtual volume method is clearly more precise on this very exotic case. Also notice that the differences between the VEM and VVM in terms of precision are very small, implying that the VVM should be preferred to the original first order VEM only for problems where conservativity matters (as the first order VEM can be extended to handle non planar faces following the technique we introduced here).

Finally, we consider the reaction dominated problem, inspired by [7]

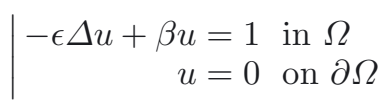

where $\Omega$ is still the unit square, in order to study the behavior of the virtual volume method in the case of vanishing diffusion tensors. The domain is subdivided as in Figure 2, and we set $\beta(\boldsymbol{x})=0.4$ for $\boldsymbol{x} \in D_{1}$, and $\beta(\boldsymbol{x})=1.0$ otherwise. When $\epsilon$ tends to zero, the solution tends to 2.5 for $\boldsymbol{x} \in D_{1}$ and 1.0 for $\boldsymbol{x} \notin D_{1}$, and presents a diffusive boundary layer. We fix the mesh, and we decrease $\epsilon$ from $10^{-3}$ to $10^{-12}$, for $\gamma=0.0,0.01$, 0.1 and 1.0. In the case where $\gamma=0$ we do not stabilize the reaction part and the "reaction fluxes" disappear. Figures 12 and 13 show the qualitative behavior of the solution, for each value of $\gamma$. We display an extrusion in the $z$ axis of the solution according to the value at the vertices, to simplify the visualization of the differences 


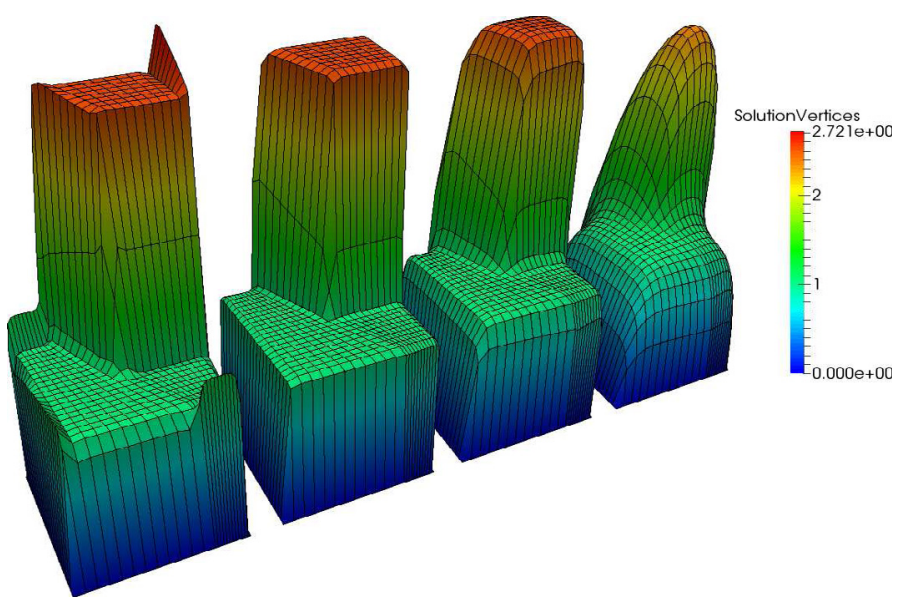

FiguRE 13. Extruded solutions for the reaction dominated case, $\gamma$ going from 0 on the left to 1 on the right, for $\epsilon=10^{-12}$.
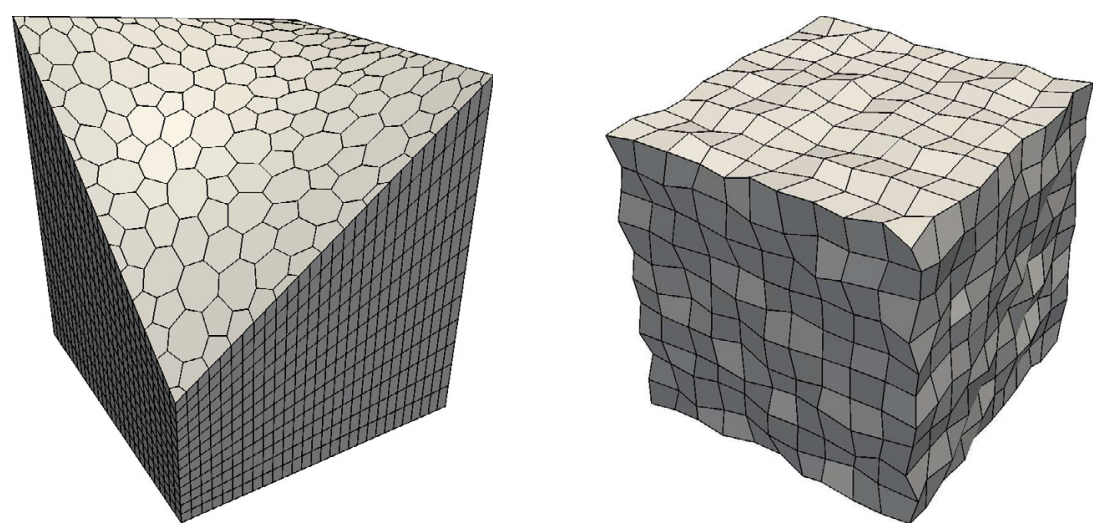

Figure 14. Example of meshes for the 3dSweep and 3dBoxRandom mesh sequences. 3dBox mesh, ConvTest5

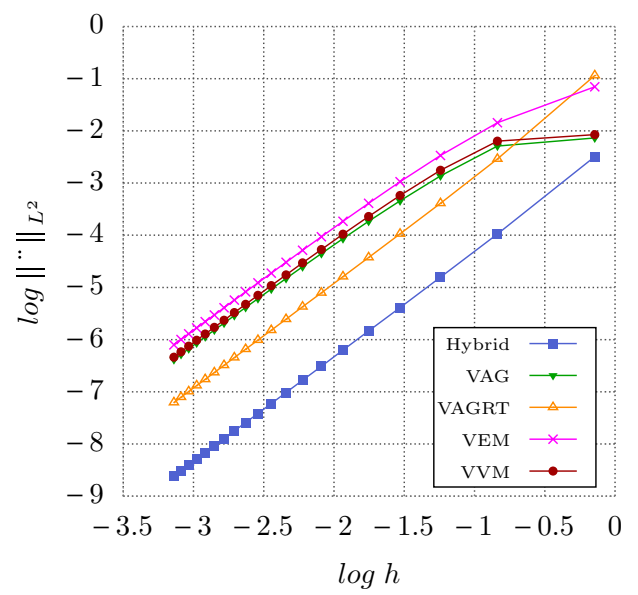
3dCheckerBoardBox mesh, ConvTest5

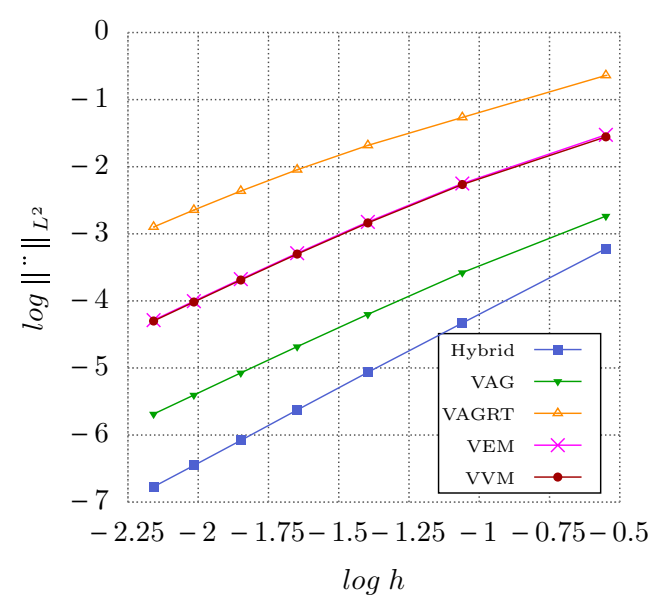

Figure 15. Convergence curves for ConvTest5 for the 3dBox and 3dCheckerBoard mesh sequences. 
between the four cases. When $\epsilon$ is small, the results are very similar, for any value of $\gamma$. However, in the same way that in [7], stabilizing the reaction part improves the behavior of the solution when $\epsilon$ becomes smaller. Also notice that the parameter $\gamma$ allows to control the size of the boundary layer.

\subsection{Numerical results in dimension 3}

We now perform a few experiments in dimension 3. To assess the behavior of the method in presence of anisotropy and on distorted mesh, we consider

$$
u(x, y)=\sin (\pi x) \sin (\pi y) \sin (\pi z) \quad \Lambda=\left(\begin{array}{lll}
\epsilon & 0 & 0 \\
0 & \epsilon & 0 \\
0 & 0 & 1.0
\end{array}\right)
$$

with $\epsilon=10^{-2}$. We test this solution on a Cartesian mesh sequence of the unit cube (named $3 d B o x$ ) and a checkerboard mesh sequence (3dCheckerBoardBox), as well as the distorted mesh sequence 3dSweep displayed in Figure 14. Meshes of this last sequence are obtained by mapping a polygonal mesh of the unit square in the $x y$ plane to a non planar surface. For the virtual volume method, the non planar faces are split by introducing a point belonging to $\widetilde{\mathcal{V}}_{\sigma}$ at the barycenter of the vertices of each face and generating triangular subfaces based on this point and the edges of the face. From the results of Figures 15 and 16, we deduce the experimental orders of convergence presented in Table 3. We see on these results that the virtual volume method reaches the optimal order in these three test cases. Our numerical experiments indicate that there is probably room for improvement in the construction of the stabilization term: we have used a very basic formula, in particular with respect to

TABLE 3. Approximate orders of convergence for ConvTest5.

\begin{tabular}{lccc}
\hline & 3dBox & 3dCheckerBoard & 3dSweep \\
\hline Hybrid & 2.00 & 2.24 & - \\
VAG & 1.90 & 1.96 & 2.06 \\
VAGRT & 2.00 & 1.72 & 1.92 \\
VEM & 1.95 & 1.95 & - \\
VVM & 1.94 & 1.95 & 2.06 \\
\hline
\end{tabular}

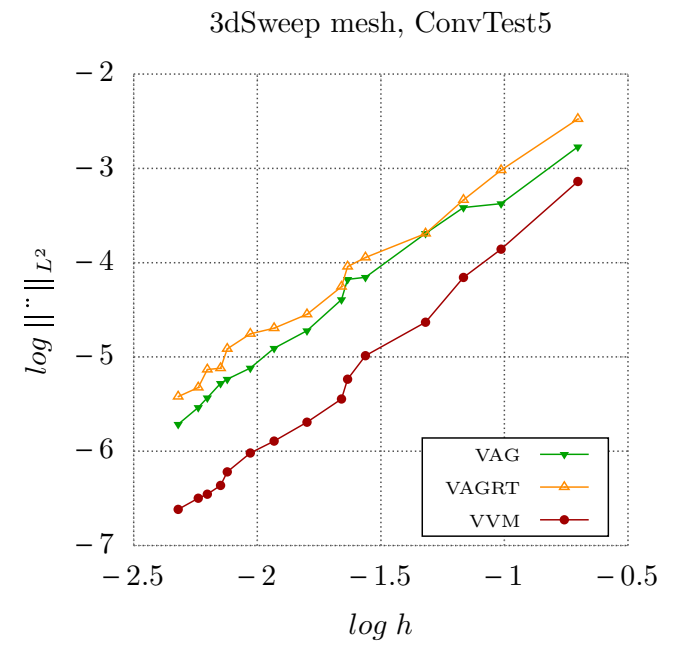

Figure 16. Convergence curves for ConvTest5 on the 3dSweep mesh sequence. 


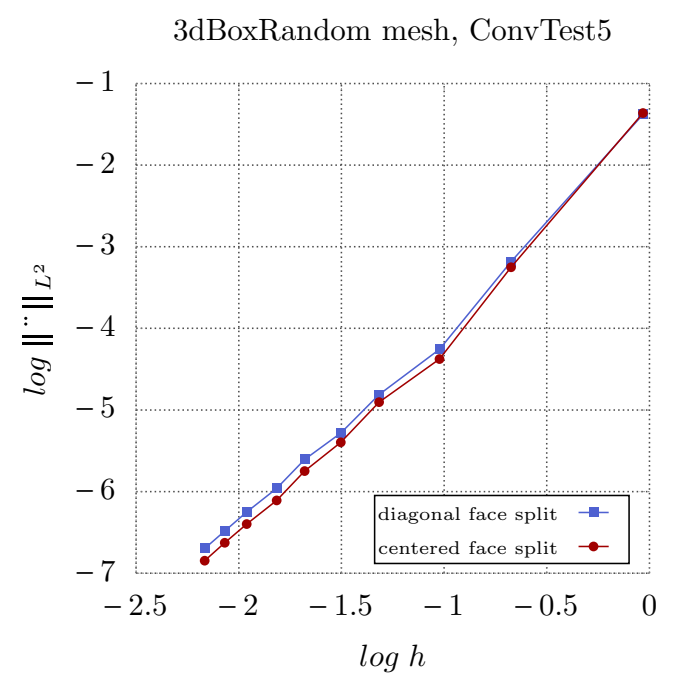

Figure 17. Convergence curves for ConvTest5 on the 3dBoxRandom mesh sequences: comparison of diagonal and centered face splitting.

the diffusion tensor, and a more involved design could probably further improve precision. Finally, to study the influence of the splitting of the faces on the solution, we consider a sequence of randomly perturbed Cartesian meshes (3dBoxRandom, see Fig. 14). On this sequence, we test two splitting strategies: the first one consist in splitting along the diagonal of each non planar squared face, which consequently gives $\widetilde{\mathcal{V}}_{\sigma}=\widetilde{\mathcal{V}}_{\partial \sigma}=\emptyset$ for any $\sigma \in \mathcal{F}$, while the second one is again based on the introduction of a point belonging to $\widetilde{\mathcal{V}}_{\sigma}$ at the barycenter of the vertices of each face, and a splitting into triangles using this point and the edges.

We clearly see in Figure 17 that this choice has very little influence on the resulting precision, thus one is completely free to choose according to practical considerations: in this particular case, it is clearly simpler to use the diagonal splitting, that evacuates the need to compute any interpolation. For generic faces, this means that an automatically generated triangular submesh of each face can be used.

\section{Conclusion}

We presented a virtual volume method based on the ideas of the VEM/mimetic technologies, keeping the classically discarded cell unknown to recover local conservativity and allow a finite volume formulation. Convergence results and error estimates were given in a general context. Numerical results in dimension 2 and 3 illustrate the good behavior and robustness of the method, even on very exotic meshes. As the method can be formulated as a finite volume method, one of its main interesting features is that its extension to more complex and general problems can follow simple, well established lines. In particular, its application to important non-linear problems such as multiphase flow in porous media is the subject of ongoing work.

\section{REFERENCES}

[1] I. Aavatsmark, T. Barkve, O. Boe and T. Mannseth, Discretization on unstructured grids for inhomogeneous, anisotropic media part i: Derivation of the methods. SIAM J. Sci. Comput. 19 (1998) 1700-1716.

[2] I. Aavatsmark, T. Barkve, O. Boe and T. Mannseth, Discretization on non-orthogonal, quadrilateral grids for inhomogeneous, anisotropic media. J. Comput. Phys. 127 (1996) 2-14.

[3] I. Aavatsmark, T. Barkve, O. Boe and T. Mannseth, Discretization on unstructured grids for inhomogeneous, anisotropic media. part ii: Discussion and numerical results. SIAM J. Sci. Comput. 19 (1998) 1717-1736.

[4] I. Aavatsmark, G.T. Eigestad, B.T. Mallison and J.M. Nordbotten, A compact multipoint flux approximation method with improved robustness. Numer. Methods Partial Differ. Eq. 24 (2008) 1329-1360. 
[5] L. Agélas and R. Masson, Convergence of finite volume mpfa o type schemes for heterogeenous anisotropic diffusion problems on general meshes. C.R. Acad. Paris Ser. I 346 (2008).

[6] L. Agélas, D.A. Di Pietro and J. Droniou, The g method for heterogeneous anisotropic diffusion on general meshes. ESAIM: M2AN 11 (2010) 597-625.

[7] B. Ahmad, A. Alsaedi, F. Brezzi, L.D. Marini and A. Russo, Equivalent projectors for virtual element methods. Comput. Math. Appl. 66 (2013) 376-391.

[8] K. Brenner, M. Groza, C. Guichard and R. Masson, Vertex approximate gradient scheme for hybrid dimensional two-phase darcy flows in fractured porous media. Finite Volumes for Complex Applications VII-Elliptic, Parabolic and Hyperbolic Problems. Vol. 78 of Springer Proceedings in Mathematics \& Statistics (2014) 507-515.

[9] S. Brenner and R. Scott, The Mathematical Theory of Finite Element Methods, 3rd edn. Springer (2008).

[10] F. Brezzi and M. Fortin, Mixed and Hybrid Finite Element Methods. Springer-Verlag, New York (1991).

[11] F. Brezzi, K. Lipnikov and M. Shashkov, Convergence of the mimetic finite difference method for diffusion problems on polyhedral meshes. SIAM J. Numer. Anal. 43 (2005) 1872-1896.

[12] F. Brezzi, K. Lipnikov and V. Simoncini, A family of mimetic finite difference methods on polygonal and polyhedral meshes. Math. Models Methods Appl. Sci. 15 (2005) 1533-1551.

[13] A. Cangiani, G. Manzini, A. Russo and N. Sukumar, Hourglass stabilization and the virtual element method. Int. J. Numer. Methods Eng. 102 (2015) 404-436.

[14] L. Beirao da Veiga, F. Brezzi, A. Cangiani, G. Manzini, L.D. Marini and A. Russo, Basic principles of virtual element methods. Math. Models Methods Appl. Sci. 23 (2013) 199-214.

[15] L. Beirao da Veiga, K. Lipnikov and G. Manzini, The Mimetic Finite Difference Method for Elliptic Problems. Springer (2014).

[16] J. Droniou, Finite volume schemes for diffusion equations: introduction to and review of modern methods. Special Edition "P.D.E. Discretizations on Polygonal Meshes". M3AS 24 (2014) 1575-1619.

[17] J. Droniou and R. Eymard, A mixed finite volume scheme for anisotropic diffusion problems on any grid. Numer. Math. 105 (2006) 35-71

[18] J. Droniou, R. Eymard, T. Gallouët and R. Herbin, A unified approach to mimetic finite differences, hybrid finite volume and mixed finite volume methods. IMA J. Num. Anal. 31 (2011) 1357-1401.

[19] R. Eymard, T. Gallouët and R. Herbin, Finite volume methods. In Techniques of scientific computiing, Part III. Handbook of Numerical Analysis, edited by P.G. Ciarlet and J.-L. Lions. North-Holland, Amsterdam (2000) 713-1020.

[20] R. Eymard, T. Gallouët and R. Herbin, A new finite volume scheme for anisotropic diffusion problems on general grids: convergence analysis. C. R. Math., Acad. Sci. Paris 344 (2007) 403-406.

[21] R. Eymard, T. Gallouët and R. Herbin, Discretisation of heterogeneous and anisotropic diffusion problems on general nonconforming meshes sushi: a scheme using stabilisation and hybrid interfaces. IMA J. Num. Anal. 30 (2010) $1009-1043$.

[22] R. Eymard, C. Guichard and R. Herbin, Small-stencil 3d schemes for diffusive flows in porous media. ESAIM: M2AN 46 (2011) 265-290.

[23] R. Eymard, C. Guichard, R. Herbin and R. Masson, Vertex-centred discretization of multiphase compositional darcy flows on general meshes. Comput. Geosci. 16 (2012) 987-1005.

[24] R. Eymard, C. Guichard, R. Herbin and R. Masson, Vertex centred discretization of two-phase darcy flows on general meshes, ESAIM: Proc. 35 (2012) 59-78.

[25] D.A. Di Pietro, Cell centered galerkin methods for diffusive problems. ESAIM: M2AN 46 (2011) 111-144.

[26] D.A. Di Pietro and A. Ern, Mathematical aspects of discontinuous Galerkin methods. Springer (2012).

[27] D.A. Di Pietro and A. Ern, Hybrid high-order methods for variable-diffusion problems on general meshes. C. R. Acad. Sci. Paris, Ser. I 353 (2015) 31-34.

[28] R. Herbin R. Eymard and C. Guichard, Benchmark 3d: the vag scheme. Springer proceedings in Mathematics, FVCA6, Prague 2 (2011) 213-222. 